\title{
Deutsche Außenpolitik nach der Vereinigung
}

Zwischen ernüchtertem Idealismus und realpolitischem Weltordnungsstreben

Die Frage nach Kontinuität und/oder Wandel deutscher Außenpolitik erweist sich als Dauerthema politikwissenschaftlicher Debatten. Der vorliegende Beitrag stellt die im Rahmen einer längeren Forschungsarbeit gewonnenen Befunde zu diesem Thema vor und kontrastiert diese mit den im Forschungsstand vorliegenden, konkurrierenden Erklärungen. Dabei wird die Leitthese entwickelt, dass deutsche Außenpolitik unmittelbar nach der Vereinigung durch einen starken normativ-idealistischen Impuls gekennzeichnet war, den sie im Laufe der folgenden Jahre angesichts der Diskrepanz von gewünschtem Welt-Soll-Zustand und realiter vorgefundenem Welt-Ist-Zustand schnell verlor und sich solcherart ernüchtert, zusehends »entidealisierte«. Zu Beginn des Beitrags wird zunächst die den Ergebnissen zugrunde liegende methodische Vorgehensweise sowie deren formaltheoretische Einbettung erklärt, um auf diese Weise auch einen Beitrag zur Reflexion interpretativer Methoden in den Internationalen Beziehungen zu leisten.

\section{Einleitung ${ }^{l}$}

Über die Frage von Kontinuität und/oder Wandel deutscher Außenpolitik entbrannte im Zuge der deutschen Vereinigung eine lebhafte Debatte zwischen Anhängern verschiedener Großtheorien der politikwissenschaftlichen Teildisziplin Internationale Beziehungen. Die Frage, ob ein vereintes Deutschland einen veränderten außenpolitischen Kurs einschlagen oder an den Grundlinien Bonner Außenpolitik kontinuierlich festhalten würde, wurde zu einem bedeutenden Austragungsort interparadigmatischer Kämpfe um innerdisziplinäre Geltung und Stellung. Als Protagonisten traten zunächst Vertreter der neorealistischen Theoriefamilie gegen jene des liberalistisch-institutionalistischen Theorienstrangs an, später erhoben sozialkonstruktivistisch argumentierende Kollegen ihre Stimmen und bildeten eine dritte Partei in diesem Streit, der im Grunde bis heute andauert. ${ }^{2}$ Denn die Frage nach einer grundlegend veränderten Identität oder grand strategy deutscher Außenpolitik er-

1 Eine frühere Version dieses Beitrags wurde im Augsburger Forschungskolloquium für Internationale Beziehungen sowie in zwei Lehrveranstaltungen des MA Sozialwissenschaftliche Konfliktforschung präsentiert und diskutiert. Mein Dank gilt allen Teilnehmerinnen und Teilnehmern, die durch ihre kritischen Rückfragen, Kommentare und Anregungen zur vorliegenden Fassung beigetragen haben. Für wertvolle Kommentare, Hinweise und Verbesserungsvorschläge möchte ich mich insbesondere bei Deniz Anan, Julika Bake, Christoph Weller, Michaela Zöhrer und den anonymen Gutachtern und Gutachterinnen bedanken.

2 Für einen Überblick über den Verlauf dieser Debatte, deren zentralen Akteure und Argumente vergleiche Roos (2010: 22-47). 
weist sich als Dauerthema wissenschaftlicher Debatte. Dies nicht zuletzt deswegen, da deutsche Außenpolitik immer wieder Anlass bot, dieser Frage mit neuer Verve nachzugehen, etwa im Zuge der Beteiligung am Kosovo-Krieg, der Nicht-Beteiligung am zweiten Irak-Krieg oder der offensiven Kampagne um einen ständigen Sitz im UN-Sicherheitsrat. Eingedenk jüngerer und jüngster Entwicklungen wird die Beschäftigung mit der Frage nach Kontinuität und/oder Wandel deutscher Außenpolitik wohl auch zukünftig ein vielbeachteter Forschungsgegenstand in den Internationalen Beziehungen bleiben (vgl. stellvertretend für die große Zahl wissenschaftlicher Reaktionen auf die deutsche Enthaltung zur UNSR-Resolution 1973 Hellmann (2011) sowie Maull (2011)).

Dem hier präsentierten Beitrag liegt ein bereits abgeschlossenes Forschungsvorhaben zugrunde, das sich vornahm, einen eigenständigen Beitrag zur Kontinuität/ Wandel-Debatte zu formulieren (Roos 2010). Dabei leiteten zwei Fragen das Forschungsvorhaben an, deren zentrale Begrifflichkeiten »Arena« und »Handlungsregel« weiter unten erläutert werden: (1) Wie haben sich die Handlungsregeln in bedeutsamen Arenen deutscher Außenpolitik von der Vereinigung bis Ende Juni 2007 entwickelt? (2) Wie haben sich die arenenübergreifenden Handlungsregeln deutscher Außenpolitik von der Vereinigung bis Ende Juni 2007 entwickelt?

Arenen bezeichnen hier bestimmte Aspekte deutscher Außenpolitik, die sich mit spezifischen issues beziehungsweise Problemen beschäftigen, beispielsweise der deutschen Europapolitik, der deutschen UN-Politik oder der deutschen NATO-Politik. Die in Frage (2) angesprochenen »arenenübergreifenden Handlungsregeln« sollen solche Handlungsregeln sein, die derart grundlegender Natur sind, dass sie für eine große Zahl oder sogar für alle außenpolitischen Arenen bedeutsam sind. Was hier unter dem Begriff »Handlungsregel« verstanden werden soll, wird in Abschnitt 2.1 des Beitrags expliziert.

Das Ziel des vorliegenden Beitrages ist es, die erarbeiteten Befunde vor einem größeren wissenschaftlichen Publikum zur Diskussion zu stellen. Dabei erscheint es sinnvoll zu sein, wenn auch möglichst knapp, zunächst die methodische Vorgehensweise zu erörtern (Abschnitt 2). Im Anschluss werden die Ergebnisse des Forschungsprozesses präsentiert und dabei systematisch mit dem Stand der Forschung kontrastiert (Abschnitt 3). Der Beitrag schließt mit einem Fazit, das an die laufende Debatte über Kontinuität und Wandel beziehungsweise Gegenwart und Zukunft deutscher Außenpolitik anschließt (Abschnitt 4).

\section{Pragmatistischer Analyserahmen und rekonstruktionslogische Methode}

Die beiden oben stehenden Forschungsfragen werden erst vor dem Hintergrund eines bestimmten formaltheoretischen Analyserahmens besser verständlich, der nachfolgend in der gebotenen Kürze dargestellt werden soll (siehe Abschnitt 2.1), bevor 
der Prozess der Datenauswahl erörtert (siehe Abschnitt 2.2.) und die methodische Vorgehensweise erläutert wird (siehe Abschnitt 2.3). ${ }^{3}$

\subsection{Formaltheoretische Grundlagen}

Wie aus den beiden oben präsentierten Forschungsfragen ersichtlich wird, stellt der erklärungsbedürftige Begriff der Handlungsregel die zentrale formaltheoretische Figur des entworfenen Analyserahmens dar. Diese Idee geht auf die Vorarbeiten des klassischen Pragmatisten Charles Sanders Peirce zurück, der Überzeugungen als Handlungsregeln (»belief is a rule for action«) begriffen hat (Peirce 1992: 129).

Jedes Handeln wird, so die Annahme, von erfahrungsgesättigten Handlungsregeln angeleitet. Dabei können Akteure aufgrund ihrer je spezifischen Erfahrungen unterschiedliche Handlungsregeln befolgen, doch diese nicht voluntaristisch, ex nihilo, aus sich selbst heraus schöpfen. Alle Handlungsregeln, so unterschiedlich sie sein mögen, sind Ergebnis sozial vermittelter Lernprozesse, die durch den Gebrauch gemeinsam geteilter Sprachzeichen kommuniziert und so intersubjektiv nachvollzogen werden können. Insofern die soziale Interaktion aller Akteure fortlaufend intendierte und nicht-intendierte Auswirkungen zeitigen, das Soziale daher genuin prozesshaft verläuft und für fortlaufende Veränderungen des größeren Bedeutungsuniversums und der gemeinsam geteilten Symbolwelt sorgt, geraten Überzeugungen immer wieder unter Bewährungsdruck. Mit mannigfaltigen Reaktionen ihrer Umwelt konfrontiert, hinterfragen bzw. reflektieren die Akteure, egal ob bewusst oder unbewusst, ihre jeweiligen Handlungsregeln fortlaufend - und zwar umso stärker und schneller, je krisenhafter die Reaktion der Umwelt auf das eigene Handeln empfunden wird. Dementsprechend modifizieren sie ihre Handlungsregeln. Die seitens einer Struktur kollektiven Handelns (Dewey 1991: 190), wie z.B. der Regierung der Bundesrepublik Deutschland verfolgten (außenpolitischen) Handlungsregeln weisen aus diesem Grund zu jedem Zeitpunkt sowohl Elemente von Kontinuität als auch Wandel auf, da sich zu jedem gegebenen Zeitpunkt einige Handlungsregeln in einer Bewährungskrise befinden und umstritten sind, während andere Handlungsregeln als bewährt gelten und als Routine ablaufen. Die propositionalen Gehalte einer Überzeugung als Handlungsregel lassen sich, so lautet eine weitere Annahme, unter Rückgriff auf interpretativ verfahrende Methoden, aus den protokollierten Spuren von Sprechakten rekonstruieren (Oevermann 1991: 295). Wer nun die Entwicklung - verstanden als Gleichzeitigkeit von Kontinuität und Wandel - des außenpolitischen Handelns Deutschlands (oder eines anderen Staates oder einer Struktur kollektiven Handelns) erklären möchte, der muss, falls ihm die-

3 Pragmatistisch inspirierte Beiträge nehmen in den Internationalen Beziehungen inzwischen an Zahl und Popularität zu (Wagner et al. 2006; Bauer/Brighi 2009; Hellmann 2009, 2010a; Friedrichs/Kratochwil 2009), gleichwohl sind davon angeleitete Forschungsarbeiten - ganz zu schweigen von Präsentationen hierdurch erarbeiteter Befunde - nach wie vor eher selten aufzufinden (vgl. jedoch Baumann 2006; Hellmann 2006; Hellmann et al. 2008). 
se theoretischen Annahmen plausibel erscheinen, die dem Handeln jeweils zugrundeliegenden Handlungsregeln im Zeitverlauf dechiffrieren. Aus dieser theoretischen Perspektive betrachtet, gilt jedes Sprechen immer bereits als Handeln, da es immer schon Reaktion auf Interaktion darstellt und auch selbst Reaktionen auslöst. ${ }^{4}$ Die diesem Handeln zugrundeliegenden Überzeugungen als Handlungsregeln lassen sich durch die Interpretation von textförmig protokollierten Spuren sozialer Interaktion rekonstruieren.

\subsection{Die Kriterien und der Prozess der Datenauswahl}

Da das Forschungsvorhaben mit dem wenig bescheidenen Ziel antrat, eine Theorie deutscher Außenpolitik, gewissermaßen ihrer grand strategy, zu entwerfen, galt es im Blick auf die methodologische Rahmung und die methodische Vorgehensweise größtmögliche Sorgfalt zu üben. Hierzu gehört auch, die Auswahl des analysierten Datenmaterials systematisch zu begründen.

Im Verlauf dreier Jahre wurden insgesamt 81 offizielle regierungsamtliche Dokumente auf darin zum Ausdruck gelangende Handlungsregeln hin analysiert und auf Grundlage dieser Rekonstruktionsarbeit eine Theorie der Entwicklung deutscher Außenpolitik von der Vereinigung bis Ende Juni 2007 entwickelt. Die Datenauswahl erfolgt dabei in Anlehnung an das theoretical sampling, demgemäß über die Auswahl des zu analysierenden Materials stets von Fall zu Fall, im Laufe des Forschungsprozesses, im Lichte der sich fortlaufend entwickelnden theoretischen Annahmen zu entscheiden sei. Für Anselm Strauss, neben Barney Glaser einer der beiden Entwickler des sogenannten grounded theory-Verfahrens, stellt das theoretical sampling einen Prozess dar, »bei dem sich der Forscher auf einer analytischen Basis entscheidet, welche Daten als nächstes zu erheben sind und wo er diese finden kann« (Strauss 1994: 70).

Das Daten-Sample wurde nicht entlang bereits im Vorfeld des Forschungsprozesses bestimmter Kriterien fixiert, sondern ergab sich als Ergebnis des Versuchs, die im Laufe der Forschung formulierten vorläufigen Interpretationen mit solchem Material zu konfrontieren, von dem anzunehmen war, dass es die bisherige Lesart möglichst maximal konzeptionell erweitern könne, um hierdurch immer erklärungskräftigere Kategorien als Elemente der entstehenden Theorie deutscher Außenpolitik zu generieren. Es wurde also von Fall zu Fall solches Material als nächster Datensatz ausgewählt, von dem zu hoffen war, dass dieser im Blick auf die sich entwickelnden theoretischen Überlegungen zur Steigerung der konzeptionellen Repräsentativität (Strübing 2002: 335) beitragen würde.

So wurde unter anderem darauf geachtet, Sprechakte verschiedener Mitglieder der Bundesregierung zu analysieren und dass neben Bundeskanzler(in) und Außenminister auch die anderen relevanten Fachminister berücksichtigt wurden. Ferner

4 Zum Verhältnis von Akteur, Struktur und Prozess aus pragmatistischer Perspektive vergleiche Franke/Roos (2010) und Roos (2010: 48-77). 
wurde darauf Wert gelegt, solche Sprechakte als Datenmaterial auszuwählen, die in verschiedenen Kontexten geäußert wurden. Genauso wichtig erschien es jedoch, besonders im Hinblick auf die zweite der beiden Forschungsfragen, also für die Rekonstruktion arenenübergreifender Handlungsregeln, zu sein, möglichst zahlreiche Grundsatzdokumente in die Analyse einfließen zu lassen, wie z.B. die jeweiligen Kapitel zur Außenpolitik der während des Untersuchungszeitraums entstandenen Koalitionsvereinbarungen $(1990 / 91,1994,1998,2002,2005)$ oder die Verteidigungspolitischen Richtlinien von 1992 und 2003.

Da die Entwicklung der grundlegenden Handlungsregeln über einen Zeitraum von 18 Jahren rekonstruiert werden sollte, galt es außerdem darauf zu achten, die Daten so auszuwählen, dass sie für den gesamten Untersuchungszeitraum repräsentativ waren, um so zu verhindern, dass die Schlussfolgerungen zu sehr von der Ausdrucksgestalt des Untersuchungsgegenstands zu einem bestimmten Zeitpunkt oder einer bestimmten Zeitspanne des größeren Prozesses bestimmt würden.

Besonders im Blick auf die erste Forschungsfrage des Projektes stellte sich das Problem, anhand welcher Kriterien sich solche Daten auswählen ließen, die eine Rekonstruktion der Handlungsregeln in »bedeutsamen Arenen« ermöglichen. Anders formuliert: Wie unterscheidet der Forscher die besonders bedeutenden von den weniger bedeutenden Arenen deutscher Außenpolitik? Insofern die rekonstruktionslogische Grundhaltung des Forschungsdesigns einer willkürlichen a priori-Bestimmung der wichtigsten Arenen deutscher Außenpolitik entgegensteht, muss die Identifikation dieser besonders grundlegenden Arenen deutscher Außenpolitik immer bereits Ergebnis des Forschungsprozesses selbst sein. Die Feinsteuerung der Datenauswahl erfolgt also je im Blick auf die sich herausbildende Theorie. Tatsächlich war es nach einem Jahr »Feldarbeit« und nach der Analyse der ersten 30 Protokolle möglich, die zentralen Arenen deutscher Außenpolitik zu identifizieren, so dass darauf aufbauend eine Feinsteuerung der Datenauswahl erfolgen konnte. Konkret bedeutet dies, dass zu diesem Zeitpunkt deutlich wurde, dass »Europapolitik« und »Sicherheitspolitik« die unangefochtenen Schlüsselarenen deutscher Außenpolitik darstellen.

Ganz im Sinne des theoretical sampling und unter steter Berücksichtigung des an grundlegenden Handlungsregeln in bedeutsamen Arenen orientierten Erkenntnisinteresses wurden in der Folge einerseits solche Dokumente analysiert, von denen im Laufe des Forschungsprozesses deutlich wurde, dass es sich hierbei um besonders zentrale Referenzen im außenpolitischen Diskursraum handelte, wie etwa die sogenannte »Humboldt-Rede« Fischers (2000a) und gleichzeitig auch solche Dokumente ausgewählt, die z.B. im Blick auf die Adressaten des Sprechaktes möglichst maximal mit den zuvor analysierten Datensätzen kontrastierten, wie zum Beispiel die Rede Fischers im selben Jahr auf der ersten Konferenz der Leiterinnen und Leiter deutscher Auslandsvertretungen in Berlin (Fischer 2000b). 


\subsection{Die methodische Vorgehensweise}

Die methodische Vorgehensweise eines Forschungsprojektes wird durch zumindest zwei Bedingungen bestimmt: Die Methode muss sich, erstens, dazu eignen, die aufgeworfene Problemstellung bearbeiten zu können und sie muss, zweitens, mit den formaltheoretischen Überzeugungen des Forschers kompatibel sein. Vor diesem Hintergrund bot sich der Rekurs unter anderem auf die Interpretation von Sprechakten zielenden sogenannten qualitativen Methoden der Sozialforschung an. Die unter 2.1 skizzierten handlungstheoretischen Prämissen des klassischen Pragmatismus übten nicht allein auf das hier präsentierte Forschungsvorhaben großen Einfluss aus, sondern auch auf zwei prominente Ansätze qualitativer Sozialforschung, nämlich das sogenannte grounded theory-Verfahren in der Variante von Anselm Strauss (1987, 1993; Strauss/Corbin 1998) und die Objektive Hermeneutik nach Ulrich Oevermann (1991, 1996, 2000). Um das Erkenntnisziel des Forschungsprojektes, eine Rekonstruktion der grundlegenden Handlungsregeln deutscher Außenpolitik nach der Vereinigung vorzunehmen, zu erreichen, wurde ein Analyserahmen gefertigt, der vor allen Dingen vom grounded theory-Verfahren, jedoch auf der Ebene der Detailinterpretation und des Dimensionalisierens der Eigenschaften des Gegenstandes zusätzlich vom Verfahren der Sequenzanalyse in der Lesart der Oevermannschen Objektiven Hermeneutik beeinflusst worden ist und der insofern einen erkennbar eigenständigen Charakter aufweist. ${ }^{5}$ Für beide Verfahren besteht sozialwissenschaftliche Forschung in der Rekonstruktion von handlungsleitenden Regelstrukturen. Oevermanns Sequenzanalyse fahndet nach sogenannten Fallstrukturgesetzlichkeiten, die er aufgrund der Rekonstruktion von bei der Auswahl von Handlungsoptionen auftretenden Mustern ableitet. Sehr ähnlich verfährt Strauss, der ebenfalls nach Regelmäßigkeiten in sozialen Interaktionen Ausschau hält, um den dahinter stehenden Handlungsregeln auf die Spur zu kommen und darauf aufbauend eine Theorie des Gegenstands zu formulieren (Strauss 1993). Obwohl auf der Ebene der konkreten methodischen Vorgehensweise zwischen Sequenzanalyse (Objektive Hermeneutik) und dem vom Memoschreiben begleiteten kontinuierlichen Vergleich und Befragen der Eigenschaften der Daten im Sinne einer »constant comparative analysis« (grounded theory) durchaus Unterschiede bestehen, überwiegen doch, gerade im Hinblick auf die systematische Berücksichtigung der Abduktion (Peirce 1965; Kelle 1995) die Gemeinsamkeiten der Ansätze. ${ }^{6}$ In einem In-

5 Die Frage der handlungstheoretischen und epistemologischen Kompatibilität dieser beiden Verfahren ist Gegenstand heftiger akademischer Auseinandersetzungen geworden (Hildenbrand 2004, 2006; Strübing 2006). Dort wird ungleich stärker das Trennende, weniger das unübersehbar Verbindende der beiden Ansätze betont.

6 Unter Abduktion versteht Peirce (unter Rückgriff auf Aristoteles) die Formulierung einer neuen Hypothese aufgrund neuer Erfahrungen. Peirce war zu dem Schluss gelangt, dass weder Induktion noch Deduktion dazu geeignet seien, neue Hypothesen zu kreieren (Peirce 1965: 89-90 (5.144)). Denn aus dem induktiven Verfahren allein resultiert noch keine modifizierte Erklärung einer bestehenden bzw. die erstmalige Formulierung einer neuen Hypothese: »Abduction is the process of forming an explanatory hypothesis. It is the only logical operation which introduces any new idea; for induction does nothing but determine a 
terview nach den "essentials « des grounded theory-Verfahrens in seiner Lesart befragt, antwortete Strauss:

»[...] Wenn ich nun sagen sollte, was zentral ist, würde ich drei Punkte hervorheben: Erstens die Art des Kodierens. Das Kodieren ist theoretisch, es dient also nicht bloß der Klassifikation oder Beschreibung der Phänomene. Es werden theoretische Konzepte gebildet, die einen Erklärungswert für die untersuchten Phänomene besitzen. Das Zweite ist das theoretische Sampling [...]. Und das Dritte sind die Vergleiche, die zwischen den Phänomenen und Kontexten gezogen werden und aus denen erst die theoretischen Konzepte erwachsen. Wenn diese Elemente zusammenkommen, hat man die Methodologie. Wie die Leute allerdings damit umgehen, hängt natürlich von ihren Bedürfnissen ab« (Strauss zitiert in Legewie/Schervier-Legewie 2004: 10-11, meine Hervorh.).

Neben dem weiter oben bereits beschriebenen theoretical sampling wurde die methodische Vorgehensweise des Projekts in der Tat maßgeblich von den beiden anderen von Strauss als essentials hervorgehobenen Elementen des grounded theo$r y$-Verfahrens, dem theoretischen Kodieren und dem permanenten Vergleich geprägt.

Theoretisches Kodieren zielt darauf ab, über eine bloße Paraphrasierung der Daten bzw. eine Katalogisierung ihrer Begrifflichkeiten hinausgehend, eine Erklärung, eine Theorie bezüglich des beobachteten Phänomens zu formulieren. $\mathrm{Zu}$ diesem Zweck wurden die in den Daten zum Ausdruck gelangenden Handlungsregeln rekonstruiert, um eine Theorie deutscher Außenpolitik formulieren zu können. Der Entwurf einer Theorie deutscher Außenpolitik basiert also auf aus dem Datenmaterial rekonstruierten Handlungsregeln. Diese Handlungsregeln werden in den allermeisten Fällen von den Akteuren nicht wörtlich ausbuchstabiert, sondern werden erst im Laufe des fortlaufenden interpretierenden Vergleichs der rekonstruierten Eigenschaften ersichtlich.

Es bietet sich an, hierzu ein kurzes Beispiel auf Grundlage einer Originalsequenz des Datensatzes zu geben, da das grounded theory-Verfahren innerhalb der deutschen IB bislang eher selten Anwendung findet:

»Vor dem Hintergrund der europäischen Integration gewinnt die Partnerschaft mit Nordamerika eine neue Qualität. In Zeiten dramatischen Wandels steht unsere in Jahrzehnten bewährte transatlantische Partnerschaft für politische Kontinuität. Für Deutschland und Europa ist der strategische Rückhalt durch die USA unverzichtbar. Und auch Amerika braucht die Unterstützung seiner europäischen Freunde für die neuen Herausforderungen. Das muss seinen Niederschlag in den transatlantischen Institutionen finden. Wir müssen die Atlantische Allianz auf die veränderten strategischen Herausforderungen ausrichten, damit sie zukunftsfähig bleibt« (Rühe 1993, meine Hervorh.).

So beantwortet der Forscher zunächst stets die Frage, auf welche Aspekte (oder Eigenschaften) deutscher Außenpolitik sich die vorliegende Sequenz bezieht bzw. welche Eigenschaften sich daraus rekonstruieren lassen. Dazu dienen ihm als Hilfs-

value, and deduction merely evolves the necessary consequences of a pure hypothesis. Deduction proves that something must be; Induction shows that something is actually operative; Abduction merely suggests that something may be. Its only justification is that from its suggestion deduction can draw a prediction which can be tested by induction, and that, if we are ever to learn anything or to understand phenomena at all, it must be by abduction that this is to be brought about« (Peirce 1965: 106 (5.171)). 
fragen: »Auf welche Aspekte/Eigenschaften deutscher Außenpolitik bezieht sich die analysierte Sequenz? Wurde bereits Material zu diesem Aspekt deutscher Außenpolitik analysiert? Wenn nein: Welche bislang noch unerforschten Eigenschaften deutscher Außenpolitik werden in der Sequenz maßgeblich thematisch?«

Diesen Fragen sehr ähnlich empfiehlt Strauss (1994: 60) dem Forscher, sich kontinuierlich die Frage zu stellen: »[A] uf welche Kategorie oder Eigenschaft von Kategorien oder auf welchen Teil der sich entwickelnden Theorie weist dieses Ereignis hin?«. Die verschiedenen Eigenschaften des Untersuchungsgegenstandes unterscheidet Strauss (1994: 51) in Kategorien. Die Sequenz wird als Ausdrucksgestalt einer oder mehrerer theoretischer Kategorien verstanden, als Komponenten einer entstehenden Theorie des Gegenstandes und diesen zugeordnet. Die Zuordnung einer jeden Sequenz zu einer bestimmten Kategorie stellt für sich bereits einen interpretativen beziehungsweise erklärenden Akt dar. Der Forscher erklärt eine Sequenz vorläufig als Ausdrucksgestalt einer (oder mehrerer) Kategorie(n). Vorläufig deswegen, da im Laufe des Forschungsprozesses ganze Kategorien aufgelöst und in eine andere Kategorie integriert werden oder aus einer alten Kategorie beispielsweise drei neue treffendere und präzisere Kategorien gebildet oder einzelne Sequenzen einer anderen Kategorie zugeordnet werden. Da zu Beginn des Forschungsprozesses noch keine einzige Kategorie oder Unterkategorie des Forschungsgegenstandes vorliegt, entstehen diese erst im Laufe des rekonstruktionslogischen Forschungsprozesses. Jede einzelne Kategorienbildung ist Ergebnis eines Interpretationsvorgangs, ist das Ergebnis der Sinnrekonstruktion der analysierten Sequenzen. Die oben stehende Beispielsequenz wurde als Ausdrucksgestalt der Kategorie »K114 Überzeugungen hinsichtlich der transatlantischen Beziehungen und den Vereinigten Staaten von Amerika interpretiert. Im Laufe des Forschungsprozesses lassen sich die einzelnen Kategorien noch weiter in verschiedene Dimensionen unterscheiden, so dass die Beispielsequenz der Kategoriendimension »K114a Europäischamerikanische Beziehungen « zugeordnet wurde und dort zusätzlich als Element der Subdimension »K114a3 Partnerschaft unter Gleichen als Zukunft der europäischamerikanischen Beziehungen; Überzeugungen mit Blick auf Fragen der gegenwärtigen und zukünftigen Ausgestaltung der europäisch-amerikanischen Beziehungen; Rekonstruktion des Westens « identifiziert wurde. An den Bezeichnungen dieser Kategorie und ihrer Subkategorien lässt sich gut zeigen, wie unter einer zunächst noch recht »breiten« Überschrift, immer spezifischere Eigenschaften einer Kategorie als Subkategorien identifiziert werden, die ihrerseits im Sinne dessen, was Strauss Dimensionalisieren nennt, die Eigenschaften der Kategorie immer spezifischer ausloten und rekonstruieren.

Der erste Abschnitt der Beispielsequenz wurde kursiv gesetzt, um zu signalisieren, dass er zusätzlich einer zweiten Kategorie zugeordnet worden ist, nämlich "K102 Überzeugungen mit Blick auf Zeit, Wandel, Prozesse, Veränderung" und dort als Ausdrucksgestalt der Dimension »K102a Grundlegender Wandel der Lage, Anpassung an neue Zeit« erklärt wurde. Es ist durchaus naheliegend, dass eine Sequenz gleichzeitig Ausdrucksgestalt ganz verschiedener Eigenschaften deutscher Außenpolitik sein kann. 
In vielen Fällen wird ein Memo verfasst, das sich der einzelnen Sequenz jeweils eindeutig zuordnen lässt. Dabei orientiert sich der Forscher beim Fertigen der Memos an einer Reihe von Hilfsfragen: Drücken sich in der Sequenz verschiedene Handlungsregeln aus - und wenn ja: In welchem Verhältnis stehen diese zueinander? Lagen die rekonstruierten Handlungsregeln bereits zuvor im Material vor und existieren weitere Memos dazu? Fügen sich die analysierten Handlungsregeln in den Bestand der bereits analysierten Handlungsregeln ein oder drückt sich hierin etwas bislang Unerforschtes aus? Welche Erklärung der Sequenz erscheint auf den »ersten Blick« als besonders plausibel? Welche Erklärungen der Sequenz lassen sich aus dem Kontextwissen des Forschers zusätzlich ableiten? Welche darüber hinausgehenden Assoziationen löst die Analyse der Sequenz aus? ${ }^{7}$

Im vorliegenden Beispiel entstand dabei folgendes Memo:

»In dieser Sequenz wird der auch bereits an anderer Stelle vertretenen Überzeugung Ausdruck verliehen, dass Kontinuität in Zeiten abrupten Wandels etwas besonders Kostbares sei, etwas Schützens- und Begrüßenswertes. Man kann getrost von einer konservativen Grundhaltung sprechen. Für die Bundesregierung symbolisiert die NATO auf besondere Weise die gewünschte Kontinuität, im Sinne von Stabilität. Ferner greift sie auf das Motiv des unverzichtbaren amerikanischen Sicherheitsbeitrages für Europa zurück, ergänzt es aber um die Idee der Wechselseitigkeit in dieser Frage, also um die Auffassung, auch Amerika bedürfe der europäischen Unterstützung. Interessant ist in diesem Zusammenhang, dass die Bundesregierung zwar einerseits die NATO als Symbol von Kontinuität ausweist, andererseits aber betont, die NATO müsse an die veränderte Lage angepasst werden. So entsteht also das Bild, dass die NATO nur dann weiterhin kontinuierlich stabilisierend wirken kann, wenn sie sich selbst der neuen Lage anpasst. Es entsteht so das Bild des Wandels als Bedingung von Kontinuität."

Das einzelne Memo dient als Ort der Konkretisierung, Präzisierung aber auch der Protokollierung des forschungsleitenden dreigliedrigen Forschungsprozesses von Abduktion, Induktion und Deduktion. Alle drei Elemente lassen sich auch im Beispielmemo finden. Offensichtlich wurden dort aus bereits zuvor rekonstruierten Protokollen drei Hypothesen abgeleitet, auf die hier induktiv Bezug genommen wird, nämlich a) dass für die Bundesregierung »Kontinuität in Zeiten abrupten Wandels etwas besonders Kostbares sei «, b) dass die NATO für die Bundesregierung »auf besondere Weise die gewünschte Kontinuität im Sinne von Stabilität« symbolisiere sowie c) dass der amerikanische Sicherheitsbeitrag für Europa aus Sicht der Bundesregierung »unverzichtbar« sei. Zusätzlich finden sich aber auch zwei abduktive Schlüsse in diesem Memo. Die aus der Sequenz rekonstruierte Überzeugung, dass aus Sicht der Bundesregierung Europa auch für die Vereinigten Staaten von Amerika unverzichtbar sei, wird hier unter dem Konzept der »Idee der Wechselseitigkeit« als neue Hypothese eingeführt. Gleiches gilt für das Konzept des »Wandels als Bedingung von Kontinuität«, das ebenfalls eine bislang unbekannte Eigenschaft des Untersuchungsgegenstandes zu erklären versucht. Die Inhalte der verschiedenen Memos werden im späteren Verlauf des Forschungsprozes-

7 Dieser Fragenkatalog bildet ein an das eigene Forschungsinteresse und die formaltheoretischen Überzeugungen angepasstes Äquivalent zu dem, was Strauss »Kodierparadigma« nennt (Strauss/Corbin 1998: 89-103). Für eine Kritik des Konzepts siehe Bryant/Charmaz (2010: 18-19):. 
ses fortlaufend miteinander verglichen. Dabei werden sowohl die bereits plausibel erscheinenden Hypothesen als auch die zunächst zaghaft formulierten neuen abduktiven Schlüsse immer weiter auf ihre Plausibilität hin überprüft, überarbeitet, reformuliert und fortlaufend durch plausiblere Erklärungen ersetzt. In einem Memo werden die Interpretationsleistungen und -ableitungen des Forschers, die Ergebnisse des permanenten Vergleichs der Eigenschaften der Daten verschriftlicht und so intersubjektiv zugänglich (Strauss 1994: 36; Strübing 2004: 83). Dabei sind die Memos auch der Ort, in dem sich Zweifel an den bisherigen Interpretationen konkretisieren und durch den fortlaufenden Vergleich mit den in anderen Memos näher dimensionalisierten Eigenschaften des Gegenstands die Überarbeitung bisheriger Hypothesen erfolgt. Die strenge Verschriftlichung ermöglicht nicht allein das Nachvollziehen der jeweiligen Interpretation der zugrundeliegenden Textsequenz, sondern schafft Transparenz bezüglich der angestellten Überlegungen im Hinblick auf vermutete Verbindungen und Zusammenhänge verschiedener rekonstruierter Handlungsregeln. Denn jedes neu formulierte Element der entstehenden Theorie muss sich in das Gesamtgefüge des bereits vorhandenen Hypothesenbestands kohärent einfügen.

Aufgrund der Vorzüge von Textverarbeitungsprogrammen ist es möglich gewesen, die Memos und das kodierte Material so anzuordnen, dass jederzeit ersichtlich blieb, auf welche Sequenzen des Datenmaterials sich welches Memo bezieht und den Überblick über die rekonstruierten Kategorien zu behalten. ${ }^{8}$ Gleichzeitig werden die an Zahl immer weiter zunehmenden Memos thematisch sortiert. Die Hauptarbeit besteht dabei in der sorgfältigen Disziplinierung des Interpretationsvorgangs. Obgleich - und hier sei eine Wiederholung ausnahmsweise gestattet - jede Wissenschaft, zumal die stärker mit Sprache als Primärmaterial arbeitende qualitative Forschung, abhängig bleibt von Interpretation, besteht die Herausforderung darin, den Interpretationsprozess intersubjektiv überprüfbar zu gestalten. Dies geschieht, indem jede Sequenz interpretiert und jede Interpretation in Form von aufeinander verweisenden Memos protokolliert wird. In den Memos wird die Interpretation der Handlungsregeln begründet und abgesichert. Indem im Laufe der Zeit immer mehr Sequenzen als Belege für bestimmte Handlungsregeln anfallen und diese systematisch verglichen werden, findet ein andauernder Prozess von Hypothesengenerierung, Überprüfung, Verwerfung und Verfeinerung statt, an dessen Ende eine kohärente, in sich widerspruchsfreie »Theorie« deutscher Außenpolitik steht. Das Ver-

8 Auf den Einsatz von spezialisierter Software der qualitativen Datenanalyse wie z.B. MAXQDA oder ATLAS.ti wurde hingegen verzichtet. Für die Zwecke der Forschungsarbeit genügten z.B. die Hyperlink-, die Inhaltsverzeichnis-, Index- und Kommentarfunktion von Word. Obwohl der Einsatz spezifischer Analysesoftware unbestrittene Vorteile mit sich bringt, wie z.B. die problemlose Integration verschiedener Dateiformate oder die farbige Markierung der in den Texten analysierten Kategorien sowie verschiedene hilfreiche Sortierfunktionen, bleibt ein Unbehagen im Blick auf die offene Frage zurück, inwiefern der Einsatz dieser Hilfsmittel die Kreativität des Interpreten dadurch beschneidet, als sie die Unmittelbarkeit des sich mit den Daten »Befassens" reduzieren und die falsche Hoffnung nähren, die Software erstelle die theoretischen Kategorien beinahe von allein (Stern 2010: 120; Hesse-Biber 2010: 327-328). 
fahren unterscheidet sich etwa von der Kategorienbildung der qualitativen Inhaltsanalyse nach Mayring (2008) insofern erheblich, als hier die Kategorien erst im Laufe der Interpretationsarbeit entstehen und nicht bereits aufgrund des gegebenen Forschungsstandes zuvor bestimmt werden. Hierdurch vergrößert sich die Wahrscheinlichkeit, bislang unbekannte Eigenschaften des Untersuchungsgegenstands in den Blick zu bekommen (Kelle 1995; Reichertz 2003; Keller 2004; Strübing 2004).

Auf diese Weise ließen sich 53 Handlungsregeln deutscher Außenpolitik rekonstruieren, die auch am Ende des Forschungsprozesses, nach zahllosen »Überprüfungen « in getrennten Interpretationsschritten und systematischen Quervergleichen ein kohärentes und plausibles Gesamtbild ergaben. ${ }^{9}$

Da die größte Zahl der Leserinnen und Leser nicht über die Zeit verfügen wird, die hier vorgelegten Interpretationen im Detail nachzuvollziehen, um auf diese Weise die Güte des Verfahrens und somit letztlich auch die Plausibilität der Interpretation beurteilen zu können, wird nachfolgend an einigen Stellen aus dem Primärmaterial zitiert, um auf diese Weise die vorgelegte Interpretation zu illustrieren. Eine solche »Belegstrategie« bleibt angreifbar, da sich auf diese Weise Interpretationen »zweiten Grades«, also solche Interpretationen, die sich erst durch den systematischen Vergleich und die Suche nach sinnlogischen Zusammenhängen von bereits rekonstruierten Bausteinen des Gesamtdiskurses ergeben, nicht belegen lassen. So gleichen die in Fußnoten mitgelieferten Belege den Photographien einzelner Bäume, aus denen je für sich genommen die sich darüber hinausgehend behaupteten Eigenschaften des ganzen Waldes nicht erkennen lassen.

Die vorliegende Arbeit tritt aber ohnehin nicht mit dem Anspruch an, die (einzig) wahre Theorie über den Forschungsgegenstand deutsche Außenpolitik zu formulieren. Denn so wenig es der pragmatistischen Logik entspricht, eine Theorie ein für alle Mal falsifizieren und durch eine überlegene Theorie ersetzen zu können, so bescheiden fallen die an die Früchte des eigenen Arbeitens geknüpften Wahrheitsansprüche aus. Aus pragmatistischer Sicht konkurrieren verschiedene Erklärungen eines Gegenstandes um die Anerkennung der wissenschaftlichen Gemeinschaft, welche über die Plausibilität - nicht über die Wahrheit oder Unwahrheit - der präsentierten Befunde unter Berücksichtigung der jeweiligen formaltheoretischen Basis und der methodischen Vorgehensweise entscheidet. Über die interne wie externe Validität und andere Gütekriterien der hier vorgelegten Theorie entscheiden also neben der Sorgfalt des Verfahrens auch immer die Leserinnen und Leser und die wissenschaftliche Debatte.

9 Aus Platzgründen können hier nicht alle Handlungsregeln tabellarisch dargestellt werden. Diese sind jedoch für einen längeren Zeitraum unter http://www.philso.uni-augsburg.de/ lehrstuehle/politik/politik1/mitarbeiter/roos/ sowie in Roos (2010) veröffentlicht. 


\section{Die grundlegenden Handlungsregeln deutscher Außenpolitik}

Im nachfolgenden Abschnitt werden die Befunde des Forschungsprozesses mit dem Forschungsstand kontrastiert und näher erläutert. Dabei wird zunächst das Leitmotiv der deutschen Europapolitik auf den Nenner des »Europäischen Deutschland in einem deutschen Europa« gebracht (Abschnitt 3.1). Daran schließt sich die Erklärung des Wandels von der Verteidigungs- zur Weltordnungspolitik an (Abschnitt 3.2). In der Folge soll die Frage geklärt werden, ob deutsche Außenpolitik sich im Zweifel stärker am nationalen Interesse orientiert oder entschlossen zur Zivilisierung der Weltpolitik beitragen möchte (Abschnitt 3.3). Am Schluss (Abschnitt 4) steht eine Antwort auf die Frage nach Kontinuität und/oder Wandel deutscher Außenpolitik, verbunden mit der Hoffnung, dass mittelfristig eine breite gesellschaftliche Debatte über die langfristigen Ziele und Wege deutscher Außenpolitik im 21. Jahrhundert geführt wird.

\subsection{Ein europäisches Deutschland in einem deutschen Europa}

Obwohl die EU immer mehr als eigenständiger außenpolitischer Akteur begriffen wird, stellt die europäische Bühne nach wie vor die zentrale Arena deutscher Außenpolitik dar. Denn die rekonstruierten Handlungsregeln machen deutlich, dass alle Bundesregierungen nach wie vor ein nationales deutsches von einem gemeinsam mit den Partnern geteilten europäischen Interesse unterschieden. Und kommt es zum Schwur, so liegt die Priorität - kaum verwunderlich - bei den enger definierten nationalen Interessen (vgl. Wessels 1999; Schmalz 2002; Janning 2006). ${ }^{10}$ Trotzdem gilt der Befund, das Verhältnis von Deutschland und Europa habe sich in ein Verhältnis von Deutschland in Europa gewandelt (Katzenstein 1997: 19), auch fünfzehn Jahre nach seiner Formulierung weiterhin. Doch trotz der fortgeschrittenen Einbettung in europäische Politiken geht damit gerade nicht der Verzicht auf eine nationale Interessenpolitik einher. Insofern ist die etwa von Hans-Peter Schwarz (2005: 272) vorgenommene Bewertung, die Bundesregierung habe die Staatsräson der Bundesrepublik durch eine Staatsräson Europas ersetzt mit den rekonstruierten Handlungsregeln nur schwer vereinbar (Goetz 1996: 40). So stimmt es zwar, dass kein Rückschritt hin zu einer nationalen Interessenpolitik feststellbar ist (Bulmer et al. 2000: 127), doch dies trifft vor allen Dingen deswegen zu, weil deutsche Europapolitik wohl immer schon Interessenpolitik gewesen ist (Paterson 2010).

$10 »$ Wir können ruhig darauf hinweisen - es wäre im Gegenteil nicht richtig, diese Tatsache $\mathrm{zu}$ verleugnen -, dass die in Kopenhagen beschlossene Erweiterung der Europäischen Union auch sehr stark im nationalen Interesse Deutschlands liegt« (Bundesregierung 2002). »Dass Deutschland europäisch denkt, heißt jedoch nicht, dass wir nur bis zum europäischen Tellerrand sehen oder die Pflege der für uns lebenswichtigen bilateralen Beziehungen vernachlässigen. Das neue Asien-Konzept der Bundesregierung zeigt, dass wir sehr wohl wissen, wo unsere Interessen liegen« (Kinkel 1995). 
Zwischen konsequenter Vertiefung und Interessenwahrnehmung besteht nämlich, wie gezeigt werden kann, kein genereller Unterschied. Die Bundesregierung fördert die Vertiefung der Integration überall dort, wo dies den nationalen Interessen Deutschlands entspricht. Wenn die deutschen Interessen durch Integration und Vertiefung nicht gefördert werden, dann entscheidet sie sich unter Verweis auf das Subsidiaritätsprinzip in der Regel dagegen. ${ }^{11}$ Die Befunde des vermeintlich neuen »contingent Europeanism《 deutscher Außenpolitik (Harnisch/Schieder 2006: 104), der »Instrumentalisierung der europäischen Ebene zugunsten deutscher Ziele« (Maull 2006a: 201), der »De-Europeanization by default« (Hellmann 2006; Hellmann et al. 2007: 39) oder der »Tendenz hin zu einer stärker pragmatisch-instrumentell ausgerichteten Europapolitik « (Gareis 2005: 224) sollten nicht als neuer Handlungsstil interpretiert werden, sondern bloß als die konsequente Anwendung einer mit dem Subsidiaritätsprinzip seit dem Vertrag von Maastricht verbundenen, Elastizität fördernden, altbewährten Handlungsregel. Denn hierin drückt sich nicht weniger aus als der kontinuierliche politische Wille, die europäische Integration nach eigenen Vorstellungen entlang nationaler Interessen zu gestalten. ${ }^{12}$

So betrachtet liegt kein Wandel der Grundhaltung deutscher Europapolitik vor, da sich diese kontinuierlich am eigenen Interesse orientierte. Vielmehr erfolgte eine Veränderung hinsichtlich der Verteilung der Fälle, wann gemeinsame Problemlösungen und wann einzelstaatliche Problemlösungen befürwortet werden (vgl. Hellmann 2006: 166). ${ }^{13}$

Statt also vom "gewachsenen Gewicht nationaler Kategorien in der deutschen Europapolitik « (Wulfert 2006: 110) auszugehen, lassen sich die beobachteten Akzent-, Stil- und Politikverschiebungen aus der hier eingenommenen Sicht primär als Resultat der kontinuierlichen Orientierung am deutschen Staatsinteresse erklären.

11 »Anders gesagt: die bisherigen Vorstellungen eines europäischen Bundesstaates, der als neuer Souverän die alten Nationalstaaten und ihre Demokratien ablöst, erweist sich als ein synthetisches Konstrukt jenseits der gewachsenen europäischen Realitäten. Die Vollendung der europäischen Integration lässt sich erfolgreich nur denken, wenn dies auf der Grundlage einer Souveränitätsteilung von Europa und Nationalstaat geschieht. Genau dieses Faktum aber steckt hinter dem Begriff der `Subsidiaritätı, der gegenwärtig allenthalben diskutiert und von kaum jemandem verstanden wird « (Fischer 2000a).

12 »Man muss sich also in das tägliche Geschäft der europäischen Verhandlungen schon im Interesse Deutschlands einmischen [...] Jeder muss in diesem Land begreifen: Wenn wir uns zusätzlich zu dem, was wir in Europa vereinbaren - das ist oft schon bürokratisch genug; das muss ich leider sagen -, Lasten aufbürden, dann haben wir gegenüber unseren europäischen Mitbewerbern keine fairen Chancen. Wir wollen aber bei aller Freundschaft zu anderen Ländern, dass in Deutschland Arbeitsplätze entstehen. Das ist die Aufgabe einer Bundesregierung. Dafür müssen wir sorgen« (Bundesregierung 2005).

13 Dabei fällt auf, dass Hellmann (2002: 28) zunächst »Europäisierung und Selbstbehauptung als die zwei Seiten ein und derselben Medaille« bezeichnet, wenige Jahre später in seiner Definition von Ent-Europäisierung dann aber die Möglichkeit der Verfolgung egoistischer nationaler Ziele innerhalb europäischer Strukturen ausschließt und für die nationale Ebene reserviert: "What do we mean by >Europeanization< and >de-Europeanization $<$ ? In our understanding, Europeanization stands for a set of beliefs (or >rules for action $<$ ) which aims for solutions at the level of EU governance structures. De-Europeanization, in contrast, denotes a similar set of beliefs which emphasizes more narrowly defined snational interests and consequently aims for solutions at the level of the nation state« (Hellmann 2006: 165). 
Ganz in diesem Sinne stellt Heinrich Schneider fest, dass in der Regel unterstellt werde, dass sowohl für den Analytiker wie im Bewusstsein der Akteure der Einsatz für eine Vertiefung der Integration und der Einsatz für spezifische nationale Interessen Deutschlands zueinander im Gegensatz stünden. Tatsächlich kann beides sozusagen »Hand in Hand« gehen. Integration kann dem Mitgliedstaat Deutschland »indirekte institutionelle Macht« zuführen und die Strategie der Einbindung könne zur »strukturellen Sicherung eigener Einflusschancen führen« (Schneider 2002: 761; vgl. Janning 2006: 748; vgl. Hellmann 2006: 164). Wenn also das Streben nach immer mehr supranationalen Handlungsregeln nach der Vereinigung nachgelassen hat, dann nicht etwa deshalb, weil innerhalb kürzester Zeit »Verantwortungspolitik« durch »Interessenpolitik« ersetzt worden wäre, sondern weil die Bundesregierung der Auffassung anhing, dass den eigenen nationalen Interessen nunmehr auf andere Art und Weise besser gedient wäre als bislang. Dies wird sowohl mit Blick auf die Integrationspolitik als auch in der Erweiterungsfrage deutlich. Sobald die frühere Losung von der »Gleichzeitigkeit von Vertiefung und Erweiterung« nicht mehr im gleichen Maße deutschen Interessen entsprach, wurde nicht allein die Integrationspolitik unter Rückgriff auf das elastische Subsidiaritätsprinzip modifiziert, sondern auch das Ende der EU-Erweiterungspolitik durch Verweise auf die Aufnahmefähigkeit der EU eingeleitet. ${ }^{14}$ Das heißt, die Bundesregierung hält zwar kontinuierlich an der Verfolgung nationaler Ziele fest, modifiziert jedoch hierbei ihre Position in zahlreichen Aspekten der Europapolitik.

Trotz dieser Veränderungen gehen die Experten davon aus, dass von einem Kurswechsel der deutschen Europapolitik »auch nicht entfernt« die Rede sein könne (Rittberger 2003: 17). Deutschland habe an einer integrationsfreundlichen Politik festgehalten, so dass sich an der Identität als »europäisches Deutschland« kaum etwas geändert habe (Risse 2004: 28). Deutschland habe weiterhin auf Integrationsfortschritte gedrängt (Maull 2006b: 274). Generell erscheint jedoch der bloße Befund des Festhaltens an Integrationsbemühungen (ähnlich wie der generelle Befund des Festhaltens am Multilateralismus) einigermaßen unspezifisch zu sein. Bedeutsamer ist, eine Antwort auf die Frage zu finden, welches Finalitätsleitbild die deutsche EU-Integrationspolitik im Kern antreibt. Was sind die mit der Integration der EU verknüpften Ziele deutscher Außenpolitik? Wie wird das Integrationsziel begründet? Besieht man die Entwicklung im Lichte dieser weitaus spezifischeren Fragestellungen, wird ersichtlich, dass sich im Laufe des Untersuchungszeitraums durchaus bemerkenswerte Veränderungen ergeben haben.

Die Entwicklung deutscher Europapolitik wird gerne entlang der Formel von entweder deutschem Europa oder europäischem Deutschland diskutiert. Die außenpo-

14 »Da wir nicht alle, die Mitglied werden wollen, aufnehmen können, werden wir die Nachbarschaftspolitik weiterentwickeln [...] Wir werden diesen Staaten eine verstärkte politische Kooperation anbieten müssen, die aber nicht in jedem Falle eine Vollmitgliedschaft bedeuten kann. Ich habe begründet warum Europa handlungsfähig bleiben muss. Ein Gebilde, das keine Grenzen hat, kann nicht in sich schlüssig handeln und eine bestimmte Verfasstheit haben. Das müssen wir uns klar vor Augen führen und deshalb Grenzen ziehen« (Bundesregierung 2006). 
litische Praxis hingegen erweist sich als deutlich kreativer, insofern die Bundesregierung beide Konzepte miteinander verbindet und - durchaus dialektisch - ein europäisches Deutschland in einem deutschen Europa anstrebt. ${ }^{15}$ Aus diesem Grund verfehlt wohl auch die These von Schwarz, der neue deutsche Sonderweg bestehe darin, Europa zu bauen und die deutsche Staatsräson zu vergessen, den Kern der Entwicklung. Statt Deutschland in Europa verankern zu wollen, wurde im Laufe der Zeit versucht, Europa in Deutschland zu verankern. Das neu entstehende europäische Gebilde sollte dem bundesdeutschen Vorbild so sehr ähneln und wäre gleichzeitig so sehr vom Einfluss des deutschen »Unionslandes« bestimmt, dass die Deutschen anstelle einer auf militärischer und wirtschaftlicher Macht basierenden zwischenstaatlichen Zwangsherrschaft nun die führende (wenigstens aber eine mitführende) Rolle innerhalb eines immer stärker föderal strukturierten Staatenverbundes innehaben würden. Gleichzeitig bliebe aber der Fortbestand der einzelnen Nationalstaaten mit ihren je unterschiedlichen nationalen Interessen und unterschiedlich gewichteten Gestaltungsmöglichkeiten garantiert (Harnisch/Schieder 2006: 105). Darin besteht der Kern des Prinzips des europäischen Deutschlands in einem deutschen Europa. ${ }^{16}$ Nationalstaatliche Souveränität soll nur in solchen Fällen auf die EU transferiert werden, in denen daraus paradoxerweise ein Nettogewinn von nationalen Gestaltungsmöglichkeiten aufgrund gemeinschaftlich realisierter Kooperationsgewinne resultiert. Angetrieben wurde das deutsche Bekenntnis zur Supranationalität von der Überzeugung, nur gemeinsam könnten die europäischen Staaten ausreichend »Gewicht« mobilisieren, um als gestaltender Pol in der neuen Weltordnung $\mathrm{zu}$ fungieren. ${ }^{17} \mathrm{Da}$ die Bundesregierung fest davon ausging, dass die vergemeinschafteten Politiken maßgeblich von deutschen Handlungsplänen bestimmt wären, erblickte sie darin weniger eine faktische Beschneidung ihrer nationalstaatlichen Souveränität als vielmehr einen Weg, um die eigenen nationalen Zielvorstellungen zur Gestaltung der Weltordnung umso effektiver umsetzen zu können. Insofern stellt die Gemeinsame Außen- und Sicherheitspolitik (GASP) die konsequent

$15 »$ Deutschland trägt aufgrund seiner Geschichte sowie seines politischen und wirtschaftlichen Gewichts eine besondere Verantwortung für den Erhalt und die Entwicklung des europäischen Integrationswerks« (Koalitionsvertrag 2005). »Damals im Jahr 2007, hat das vereinte Europa nach kräftezehrenden und nervenaufreibenden Diskussionen am Ende die Weichen richtig gestellt; damals, im Jahr 2007, da hat die Europäische Union den richtigen Weg in eine gute Zukunft eingeschlagen. Das war, ist und bleibt unser Auftrag, unser Auftrag für die Zukunft. Deutschland wird sich dafür auch nach seiner Präsidentschaft mit ganzer Kraft einsetzen« (Merkel 2007).

16 Dieser Befund ähnelt beinahe wörtlich den Überlegungen zur Gleichzeitigkeit von »uploading«- und »downloading«-Prozessen deutscher EU-Politik, wie sie z.B. Paterson (2010: 45) in seinem jüngsten Beitrag zur Debatte formuliert: »In the immediate post-Wall years a German vocation for Europe appeared to have reached its apogee in terms both of a more European Germany, the traditional aim of German European policy, and increasingly in a second (and more novel) sense of a German Europe where German preferences were uploaded to a European level«.

17 »Nur wenn wir gemeinsam und gezielt handeln, haben wir Europäer Aussichten, unsere Anliegen in der Welt zur Geltung zu bringen [...] Eine kohärente Außenpolitik und das Sprechen mit einer Stimme werden jeden Tag wichtiger für ein Europa, das sich in der Welt behaupten will« (Merkel 2007). 
machtpolitisch kalkulierte Antwort auf ein multipolares Weltmodell dar und kein genuin idealistisch gespeistes Vergemeinschaftungsprojekt. Diese Grundhaltung diversifizierte sich durch die neu entstandenen Konzepte des Wiedererstarkens des Nationalstaats und des effektiven Multilateralismus noch zusätzlich. Wenn die Bundesregierung davon ausging, dass das einzelstaatliche Potential Deutschlands im Verbund mit anderen großen Mächten ausreiche, um die gewünschten Resultate zu erzielen, etwa bei der P5+D-Initiative oder bei der G4-Reforminitiative des Weltsicherheitsrats, schwächte sich das Bekenntnis zu supranational-europäischen Gewinnmaximierungsstrategien zugunsten einer einzelstaatlich-egoistischen Gewinnrealisierungsstrategie deutlich ab. ${ }^{18}$

\subsection{Von der Verteidigungs- zur Weltordnungspolitik}

Bis zur Vereinigung galt die feste Einbindung in die westliche Wertegemeinschaft als das unangefochtene Leitmotiv deutscher Außenpolitik. Zwar ist auch für die Zeit nach der Vereinigung generell festzustellen, dass die deutsche Außenpolitik an westlichen Werten orientiert bleibt (Erb 2003: 3; Hellmann et al. 2006: 104), doch der Westen als spezifische zwischenstaatliche Vergemeinschaftungsform ist nach 1989/90 kaum noch thematisch. Stattdessen setzt sich immer stärker das Bild zweier konkurrierender Pole (EU und USA) in einem multipolaren Rahmen durch. ${ }^{19}$ Nicht nur unterscheidet sich das Europa der 27 von der früheren maßgeblich westeuropäisch geprägten EU-15 und unterliegt insofern genauso stark östlichen wie westlichen Einflüssen, sondern es stellt inzwischen auch mehr als bloß den europäischen Pfeiler des westlichen Bündnisses innerhalb der US-geführten westlichen Wertegemeinschaft dar. Die Bundesregierung versteht die EU und die USA als zwei in letzter Konsequenz getrennte Wertegemeinschaften mit unterscheidbaren Identitäten, die zwar miteinander viele Überzeugungen und Prinzipien teilen, deren Bestand und Gehalt, wenn auch ähnlich, so doch keineswegs identisch sind. ${ }^{20}$ Der »Westen« als an den Ost-West-Konflikt gebundene ordnungspolitische Figur hat an Bindekraft deutlich eingebüßt und wurde stattdessen von der Idee des multipolaren

18 Dies fügt sich in jene Beobachtung, die einen Bedeutungsgewinn intergouvernementaler zu Lasten supranationaler Leitmotive in der deutschen Europapolitik diagnostiziert (Göler 2004: 134).

19 »In der multipolaren Welt des 21. Jahrhunderts muss die Europäische Union deshalb zum eigenständigen, politisch handlungsfähigen Subjekt werden« (Fischer 1999a). »Manchmal freut man sich auch in Amerika darüber, wenn die Europäische Union ein paar Probleme mit sich selbst hat [...] Aber ich glaube, dass wir uns in den nächsten Tagen werden entscheiden müssen. Ist der große Kampf der zwischen Boeing und Airbus? Oder ist die große Frage die, wie wir gemeinsam mit unseren Werten in der Frage des Umgangs mit China umgehen? (Merkel 2006).

20 »Deshalb müssen wir darüber nachdenken, was Europa bedeutet und wie der Gestaltungsanspruch der Politik wieder durchgesetzt werden kann. Viele haben den Eindruck, dass es hier nur um den Fluss von Kapitalströmen geht, dass die Politik gar keine Kraft mehr hat. Wir müssen aber unsere Überlegenheit zeigen. Daher ist es, glaube ich, richtig und wichtig, dass wir sehen: Mit 450 Millionen Menschen in der Europäischen Union können wir natürlich die Regeln des Welthandels beeinflussen« (Bundesregierung 2006). 
Weltsystems ersetzt, in der die EU als Ganzes zwar nach wie vor westlichen Werten verpflichtet bleibt, jedoch nicht mehr länger als Teil einer monolithischen westlichen, den Atlantik überspannenden Gemeinschaft gedacht wird, sondern als von den USA und der NATO unabhängiger, eigenständiger Pol agiert. Entsprechend soll auch den von den deutschen Werten maßgeblich bestimmten europäischen nicht den westlichen - Werten zu einer weltweit führenden Rolle verholfen werden. Insofern steht die Feststellung von Schwarz (2005: 274), die rot-grüne Bundesregierung habe das Fernziel angestrebt, aus Europa eine Weltmacht zu machen, durchaus im Einklang mit der hier vorgelegten Interpretation. Dieses Ziel stellt jedoch spätestens seit 1993 eine Konstante deutscher Außenpolitik dar - unabhängig von den beiden Regierungswechseln 1998 und 2005 und zumindest bis zum Ende des Untersuchungszeitraums. Gunther Hellmann hat wohl aus einer binneneuropäischen Sicht Recht, wenn er in der europäischen Integration sowohl ein Vehikel der deutschen Resozialisierung und des machtpolitischen Aufstiegs als auch ein Mittel gegen die »Übel des Nationalismus« und der »deutschen Machtstaatstradition« erblickt (Hellmann et al. 2006: 212); doch angesichts der rekonstruierten Handlungsregeln droht eine Übertragung der innerhalb Europas weitgehend eingehegten und kanalisierten Mächtekonkurrenzlogik auf das Weltsystem. ${ }^{21}$ Schon Link (2004a: 3) und Pradetto (2006: 25) hatten auf die Ankündigung einer »Europe puissance« im Rahmen einer multipolaren Welt als bedeutsame Entwicklung deutscher Außenpolitik hingewiesen. Mit Blick auf die zentrale Annahme der Bundesregierung, die Zukunft der internationalen Beziehungen werde durch eine multipolare Systemstruktur bestimmt, stellt sich die Frage, welcher Art dieses multipolare System letztlich sein soll. Insofern die Unterscheidung von Innen und Außen für Außenpolitik konstitutiv ist (Hellmann et al. 2007: 21), gleichzeitig aber deutsche Außenpolitik das Innen anderer Staaten immer weniger anzuerkennen bereit ist und stattdessen die Intervention in deren Angelegenheiten aufgrund von Interdependenzverweisen im Sinne eines Interventionsanrechts zur Regel erhebt und gleichzeitig Eingriffe von außen in die eigenen oder europäischen Angelegenheiten kategorisch ablehnt, steht eine Begrenzung des Souveränitätsprinzips auf einige wenige Mächte zu Lasten der überwältigenden Zahl der Staaten bevor. ${ }^{22}$ Gerade die Analyse des

21 »Das heutige internationale System wird nicht mehr von Blöcken bestimmt, sondern von einer Vielzahl von Staaten « (Bundesregierung 2004). »China mit einem Viertel der Weltbevölkerung und Indien mit fast einer Milliarde Menschen sind heute Schwergewichte in der Weltpolitik. Und die kleinen und mittleren Staaten schließen sich nach dem Vorbild der Europäischen Union zu regionalen Gruppierungen wie ASEAN oder MERCOSUR zusammen. Mit NAFTA und APEC sind unter Führung der USA Zusammenschlüsse entstanden, die die bevölkerungsreichsten Kontinente umfassen« (Kinkel 1998).

22 »Allein im letzten Monat kamen 1500 Flüchtlinge aus dem Kosovo. Wir haben mit die Hauptlast zu tragen. Völlig unabhängig von der Frage der Stabilität der Region hat Deutschland ein Anrecht darauf, Herrn Milosevic zu sagen: Dies ist nicht eine innere Angelegenheit ihres Landes. Vielmehr haben wir Deutschen daran, auch wegen der BosnienFlüchtlinge, zu Recht ein besonderes Interesse. Das müssen Sie schon zur Kenntnis nehmen« (Bundesregierung 1998). »Das aus der Souveränität der Staaten abgeleitete Verbot, sich in die inneren Angelegenheiten eines anderen Staates einzumischen, ist wohlbegründet. Aber staatliche Souveränität ist nur ein Rechtsgut. Die Souveränität der Staaten findet ihre Grenzen in dem völkerrechtlichen Gebot, die elementaren Menschenrechte zu ge- 
Konzepts des effektiven Multilateralismus und der damit einhergehenden stärkeren Betonung einzelstaatlicher Regelungskompetenzen hat gezeigt, dass in diese neue Form des Multilateralismus nur noch einige wenige besonders mächtige Staaten beziehungsweise staatsähnliche außenpolitische Mächte einbezogen sein werden. So entsteht ein Bild, das die Verhältnisse des multipolaren europäischen Mächtegleichgewichtsystems nach 1648 bis 1945 im Rahmen einer allgemeinen Fokusverlagerung weg vom europäischen und hin zum globalen Machtgleichgewichtsdenken als Schablone auf die Welt überträgt. Aus dieser auf der Multipolaritätsannahme begründeten geopolitischen Perspektive heraus erscheinen einige wenige große Mächte als die bestimmenden Einheiten im Weltsystem, sei es im Rahmen eines »effektiven Multilateralismus« oder weniger optimistisch betrachtet als Kontrahenten im Kampf um Ressourcen und kulturellen Einfluss in der Welt. Das in den Weltzukunftsbeschreibungen deutscher Außenpolitik immer prominenter platzierte Konzept der Multipolarität greift jedenfalls unmittelbar auf die Ideen der Konkurrenz zwischen einigen wenigen großen Mächten beziehungsweise der bestehenden Interessengegensätze sowie das damit verknüpfte Konzept des Machtgleichgewichts zurück. ${ }^{23}$ Einem physikalischen Gravitationskonzept entsprechend sollten jene Regionen und Staaten, die selbst keinen handlungsfähigen Pol in diesem Weltsystem darstellen, möglichst eng an die EU gebunden und in das eigene Kraftfeld integriert werden. Die GASP der EU zielt im Kern auf die Gestaltung der Welt entlang europäischer Vorstellungen und Ideen. Dabei ging die Bundesregierung von der Überlegenheit der europäischen Werte - die ihrerseits vor allen Dingen von deutschen Vorstellungen geprägt werden - gegenüber den konkurrierenden Werten anderer Mächte (auch der USA) aus. ${ }^{24}$

Diese Weltsicht basiert jedoch trotz der zentralen Stellung des europäischen Staatenverbundes gerade nicht auf der früher dominanten Idee der Schwäche der Nationalstaaten, sondern ganz im Gegenteil auf der Idee der überlegenen Stärke einiger weniger außenpolitisch handlungsfähiger, sich zueinander trotz weltweiter Interdependenz dennoch weitestgehend autonom verhaltender Mächte. ${ }^{25}$ Die von Bernhard Zangl und Michael Zürn (2003) beschriebene, stark normativ konnotierte postnationale Konstellation, in der die klassische machtstaatliche Außenpolitik ausgedient habe, erscheint, aus dieser Perspektive betrachtet, zu früh postuliert worden zu sein. Machtstaatliche Außenpolitik bestimmt weiterhin das Weltgeschehen und beinahe genauso wie vor dreihundert Jahren ist die Zahl der daran gestalterisch partizipier-

währleisten und das Selbstbestimmungsrecht der Völker zu achten. Diese drei hohen Rechtsgüter haben gleichen Anspruch auf Respekt (Scharping 1999).

23 Als gutes Beispiel für die Zentralität des Konzepts der Ressourcenverteilung kann die deutsche Russlandpolitik dienen. Angesichts der aufgezeigten Entwicklung wird deutlich, dass - abhängig von der Einschätzung der russischen Ressourcen - die deutsche Rollenbeschreibung Russlands von der Weltmacht zum Entwicklungshilfeobjekt zurück zur Großmacht variierte.

24 Vgl. Bundesregierung (2006) in Fußnote 20.

25 Außer der EU handelt es sich bei den von Deutschland identifizierten Weltpolen tatsächlich stets um Nationalstaaten: Neben den USA und Russland gelten inzwischen auch China und Indien als Pole. 
enden Akteure auf eine geringe Zahl begrenzt. Folgerichtig hat die bereits 1991 von Klaus Dieter Wolf angestellte Beobachtung, der gemäß gerade die »Annahme von der Kontrollierbarkeit und Steuerbarkeit der internationalen Politik durch die Machtmittel des Staates aber - die Vorstellung von Macht als Ordnungsfähigkeit« überholt sei (Wolf 1991: 248), als Handlungsregel ihren Einfluss auf deutsche AuBenpolitik zusehends zugunsten der Idee der wiedererstarkten einzelstaatlichen beziehungsweise EU-europäischen Problemlösungskompetenz eingebüßt. Ganz in diesem Sinne zeigen zum Beispiel die deutsche Position zur Reform des Weltsicherheitsrats, die im Rahmen der P5+Deutschland-Initiative eingenommene Rolle und auch die Enthaltung zur Libyen-Resolution RES 1973 des UNSR, dass die Bundesregierung Deutschland inzwischen die Rolle einer Weltmacht zuschreiben möchte, die, falls es der deutschen Interessenlage entspricht, auch unabhängig von den Positionen der EU- und NATO-Partner entscheidet.

\section{Der Wandel der Sowohl-als-auch-Politik: Die neue deutsche NATO-Politik}

Das Konzept der deutschen Sowohl-als-auch-Politik beschreibt seit jeher eine Strategie, die auf eine vermittelnde Position zwischen Frankreich (EU) und den USA (NATO) zielt. In den letzten Jahren diagnostizieren immer mehr Studien die Auflösung dieses grundlegenden Bausteins deutscher Außenpolitik zu Gunsten eines unübersehbaren Vorrangs der EU gegenüber den transatlantischen Beziehungen. ${ }^{26}$ Obwohl die rekonstruierten Handlungsregeln diesen Befund generell bestärken, erweist er sich in seinen Elementen als überraschend komplex.

Denn die deutsche Strategie versucht die Sowohl-als-auch-Politik insofern fortzuschreiben, als sie zwar dem Leitsatz in dubio pro Europa (Link 2004a) folgt, jedoch sowohl auf das Werkzeug der Europäischen Sicherheits- und Verteidigungspolitik (ESVP) setzt als auch den Einfluss Europas auf das NATO-Instrumentarium zu vergrößern sucht (vgl. Weiss 2009: 337). Die stärkere Betonung europäischer Zwecke geht also keineswegs mit einer Abkehr von der NATO einher, sondern führt zu der Idee, dass die europäische Handlungsfähigkeit innerhalb der NATO gestärkt werden müsse (vgl. Knapp 2004: 170). ${ }^{27}$ Dies fügt dem bisherigen einhelligen Befund der Experten hinsichtlich des Festhaltens an der NATO ${ }^{28}$ eine neue Erklärung hinzu: Deutsche Außenpolitik zielte weniger auf eine Emanzipation von der NATO, sondern nach mehr Spielraum durch größere Unabhängigkeit von den

26 Maull (2004: 18); Schöllgen (2004: 9); von Bredow (2006: 246-247); Miskimmon/Paterson (2006: 42).

$27 »$ Die NATO ist der stärkste Anker unserer Sicherheits- und Verteidigungspolitik. Wir setzen uns dafür ein, dass die Atlantische Allianz zum zentralen Ort des transatlantischen sicherheitspolitischen Dialogs wird, an dem die transatlantischen Partner ihre strategischen Vorstellungen gleichberechtigt konsultieren und koordinieren. Auch dadurch wollen wir die Allianz stärken. Auf enge und reibungslose Abstimmung zwischen NATO und EU gilt es dabei zu achten « (Koalitionsvertrag 2005).

28 Katzenstein (1997: 32); Le Gloannec (2004: 31); Knapp (2004: 170); Hellmann et al. (2006: 105); Miskimmon/Paterson (2006: 37). 
USA (vgl. Gareis 2005: 223). Der Fortbestand der NATO bleibt ganz in diesem Sinne ein kontinuierliches Ziel deutscher Außenpolitik. Generell soll die NATO jedoch nicht mehr länger ein US-geführtes Unternehmen bleiben, sondern den Partnern auf beiden Seiten des Atlantiks gleichberechtigt zur Verfolgung gemeinsamer, aber auch ihrer jeweils spezifischen Interessen zur Verfügung stehen. $\mathrm{Zu}$ diesem Zweck soll die Position Deutschlands und der EU innerhalb der NATO-Strukturen möglichst umfassend gestärkt werden. Angesichts dieser stringenten deutschen NATO-Strategie verfehlt die Kritik von Schwarz (2005: 30), der einen neuen Wankelmut in der deutschen Sicherheitspolitik in Form eines steten Vor und Zurück, eines permanenten Hin und Her zwischen NATO-Kooperation und dem Bemühen um eine autonome europäische Verteidigungsstreitmacht festgestellt haben will, den neuralgischen Punkt: Die deutsche Sicherheitspolitik ist nicht »in der Hamstertrommel der Widersprüche herumgesaust« (Schwarz 2005: 30), sondern agiert nach einem ganz rationalen Kalkül und drängt dabei genauso nach größtmöglichem Einfluss auf die NATO wie sie gleichzeitig die Handlungsfähigkeit der ESVP vorantreibt.

\section{Die Bundeswehr als Instrument deutscher Weltordnungspolitik}

Besonders der Transformationsprozess der früheren Verteidigungs- hin zur heutigen Weltordnungspolitik macht den grundlegenden Wandel deutscher Außenpolitik auch für den politikwissenschaftlichen Laien offensichtlich. Doch angesichts der Zielstrebigkeit, mit der die Bundesregierung die Ermöglichung des Einsatzes deutscher Streitkräfte im Ausland bereits unmittelbar nach dem Ausscheidens HansDietrich Genschers bis zum BVerfG-Urteil im Juli 1994 verfolgte, erscheint es nicht nur aus heutiger Perspektive rätselhaft, wie damals konstatiert werden konnte, Deutschland führe seine Politik der Unterbetonung militärischer Instrumente beim Verfolgen nationaler Ziele weiter (Berger 1996: 317; 1998: 208). ${ }^{29}$ Auch den im Blick auf die Analyse deutscher Außenpolitik bis heute diskursbestimmenden Hanns W. Maull (1992: 271) stellt gerade der Versuch, das Konzept der militärischen Zurückhaltung »mit Ausnahme von individueller und kollektiver Selbstverteidigung « als wichtigen Teil der eigenen Zivilmachtsthese mit der zunehmenden Zahl und Intensität militärischer Einsätze in aller Welt zu vereinbaren, offensichtlich vor Schwierigkeiten. Trotz der veränderten Einsatzpraxis geht er nach wie vor davon aus, dass sich Deutschland »ohne jeden Zweifel« seinen Skeptizismus hinsichtlich des Einsatzes militärischer Mittel zur Problemlösung bewahrt habe (Maull 2006b: 274). Unklar bleibt, wie sich das Konzept der Armee im Einsatz, das den Einsatz zum Normalfall erklärt, mit den Befunden vom Fortbestand der traditionellen Zurückhaltung im Gebrauch militärischer Mittel (Erb 2003: 3) oder dem Be-

29 Auch die Behauptung Katzensteins (1997: 43), Deutschland sei im Unterscheid zu Frankreich und Großbritannien kein militärischer Akteur, dürfte zwischenzeitlich als widerlegt gelten. 
fund der beinahe irrationalen Zurückhaltung beim Einsatz von Gewalt im Äußeren (Müller 2006: 63) beziehungsweise der unveränderten Zögerlichkeit beim Engagement in den klassischen außen- und sicherheitspolitischen Politikfeldern (Gareis 2005: 225; Maliki 2006: 39) verträgt.

Inwieweit die allgemeine »Kriegs- und Militärmüdigkeit« als ungebrochene Konstante der politischen Kultur Deutschlands (Krippendorff 2010: 91) und die beharrliche Bevölkerungspräferenz, im Gegensatz zu den politischen Entscheidungsträgern, nur sehr begrenzte Einsatzszenarien zu unterstützen (Göler 2010: 197), die veränderten Handlungsregeln der Bundesregierung zukünftig wirksam beschränken kann oder sich die pazifistische Grunddisposition der deutschen Gesellschaft schleichend der veränderten Einsatzrealität anpassen wird, ist eine empirisch offene Frage.

Tendenziell schließt sich der Verfasser aufgrund der eigenen Rekonstruktionsarbeit eher der Situationsbeschreibung Werner Links (2004b: 49) an, demzufolge der "politische Pazifismus« hinweg gefegt worden sei - und nicht der These Müllers (2006: 64), die deutsche Zurückhaltung im Irak-Konflikt 2002 sei durch die Priorität des Pazifismusprinzips erklärbar. ${ }^{30}$ Auch Pradetto (2006: 24) erkennt die größten Veränderungen in der seiner Meinung nach ansonsten stabilen Zivilmachtsrolle mit Blick auf die Grundhaltung zur Frage des Einsatzes der Streitkräfte, was sich durchaus darin spiegelt, dass Deutschland inzwischen zu einem der größten internationalen Truppensteller avanciert sei (Wagener 2006: 83). Die Erklärung von Longhurst (2004: 145), der grundlegende Wandel der deutschen Haltung zum Einsatz der Bundeswehr sei Resultat der Bemühungen, weiterhin als möglichst zuverlässiger Partner zu gelten, nimmt wohl nur einen bestimmten Teilausschnitt des Begründungsmuster in den Blick. Der Wunsch, »als verlässlich zu gelten«, stellt sich in der größeren Perspektive nicht als das zentrale Ziel dar, sondern als begünstigender Faktor eines weit grundlegenderen Motivs. Als weit wichtiger erweist sich nämlich die Überzeugung, dass die deutsche Beteiligung an Militärmissionen die grundlegend notwendige Bedingung für die deutsche Sicherheitspolitik als an den nationalen Interessen orientierte, eigenständige Weltordnungspolitik darstellt. Im Rahmen dieser mit den höchsten Gestaltungsansprüchen verknüpften Weltordnungspolitik symbolisieren Militäreinsätze Verantwortungsübernahme und werden inzwischen wie selbstverständlich - und insofern eben gerade nicht mit der größtmöglichen Zurückhaltung - als Tauschware für Ansehen und Gestaltungsansprüche eingesetzt. Das Konzept der Armee im Einsatz ist das folgerichtige Ergebnis des deutschen Anspruchs auf eine führende Gestaltungsrolle in der Welt und ein sichtbares Zeichen dafür, wie stark das Zivilmachtkonzept deutscher Außenpolitik inzwischen an den auch von Maull (2011: 112) selbst beschriebenen »Abnutzungserscheinungen« leidet.

30 Trotz des Befundes der weitgehenden Routinisierung des Kämpfens und Sterbens im Rahmen des »Armee im Einsatz«-Konzeptes geht der Verfasser jedoch nicht so weit wie Schwarz (2005: 306), der von einem Wandel vom »Quasi-Isolationismus« hin zum »leichtfüßigen Interventionismus« spricht. 


\subsection{Grundlegende Machtorientierung: Nationales Interesse oder Zivilisierung der Welt?}

Im Blick auf das Selbstverständnis deutscher Außenpolitik bezogen auf die eigene Macht und den Umgang der Bundesregierungen mit dieser, steht weniger die Frage im Mittelpunkt, $o b$ deutsche Außenpolitik nach Macht strebt und diese zur Verfolgung eigener Interessen einsetzt oder nicht. Im Kern der wissenschaftlichen (und gesellschaftlichen) Auseinandersetzung der letzten Jahre steht vielmehr das Problem, für welche Zwecke die deutsche Macht eingesetzt wird und wie dies von der Exekutive begründet wird. Das vorherrschende wissenschaftliche Narrativ zur deutschen »Machtfrage« lässt sich wohl so zusammenfassen, »dass von einer Wende hin zu einer an Prinzipien der Machtgewinnung orientierten Politik keine Rede sein könne« (Risse 2004: 24).

Und tatsächlich kann von einer wie auch immer gearteten »machtpolitischen Wende« deutscher Außenpolitik wirklich keine Rede sein. Dies vor allen Dingen deswegen, weil das Streben nach größerer Macht im Vergleich zu anderen Staaten beinahe während des gesamten Untersuchungszeitraums (mit Ausnahme der ersten beiden Jahre) eine Konstante darstellte. Das Ziel, die eigene Macht durch Effizienzoptimierung immer weiter in Konkurrenz mit anderen Staaten zu steigern, gewann im Laufe des Untersuchungszeitraums immer mehr an Bedeutung. Die Bundesregierungen orientierten ihre Handlungspläne nicht nur entlang der Analyse der internationalen Machtverteilung in Anlehnung an die Hypothesen der (neo-)realistischen Schule, sondern waren außerdem an relativem Machtzuwachs durch Effizienzsteigerung in Konkurrenz zu anderen Staaten interessiert. ${ }^{31}$ Schließlich zeigten sich die Bundesregierungen davon überzeugt, dass dem deutschen Gemeinwesen und Staatsapparat ein besonderer Effizienzgrad eigen sei, der als Faktor das objektive Machtpotential in Relation zu anderen, vielleicht mit größeren Potentialen aber geringerer Effizienz versehenen Konkurrenten, erheblich steigert.

Die deutsche »Zivilmacht« (Maull 1992) strebte zwar nach mehr Verantwortung bei der Verregelung der internationalen Beziehungen, doch dies bedeutete gerade nicht den Verzicht auf klassische Machtpolitik und deren militärische Instrumente, sondern zielte auf ein Mehr an nationaler Gestaltungsmacht im Vergleich und in Konkurrenz zu anderen Staaten unter Anpassung der traditionellen Mittel an die modernen Bedingungen. Innerhalb eines sehr kurzen Zeitraums von maximal zwei Jahren nach der Vereinigung verloren die Konzepte von Verantwortung aufgrund

$31 »$ Wir wollen für eine abgestimmte und effiziente Vertretung deutscher Interessen in den europäischen Institutionen sorgen« (Koalitionsvertrag 2005). Es ist schon überraschend, dass das Effizienzkonzept in den analysierten Protokollen des Zeitraums 1990-93 nicht ein einziges Mal Erwähnung fand, es aber immer häufiger thematisch wurde, je weiter die Zeit voranschritt. Dies erstaunt gerade deswegen, weil die Bundesregierung schon früh auf die "gewaltigen Ausgaben« hingewiesen hatte (Genscher 1990), vor denen Deutschland aufgrund der Vereinigung stünde. So hätte man eigentlich gerade in den ersten Jahren nach der Vereinigung die Verankerung des Effizienzkonzepts in den außenpolitischen Handlungsregeln erwarten müssen. Dies erfolgte aber erst viel später, beschleunigte sich dann aber zusehends. 
der eigenen Geschichte beziehungsweise von Verantwortung als selbstverständlicher und selbstloser Beitragsverpflichtung aufgrund der eigenen Potentiale an Relevanz. Sie wurden durch die Konzepte der Verantwortung als Anspruch auf Mitsprache aufgrund der eigenen Beiträge sowie dem Konzept des Ansehenserwerbs durch Verantwortungsübernahme ersetzt. ${ }^{32}$ Damit geht ein Trend einher, verstärkt eigene Erwartungen gegenüber der internationalen Umwelt zu benennen. Das explizite Formulieren von Erwartungen signalisiert dabei, dass ein Anrecht darauf besteht, das Handeln des Adressaten entsprechend der eigenen Vorstellungen zu beeinflussen. Dies stellt ein zusätzliches Element der neuen deutschen Weltordnungspolitik und der daran gebundenen Führungsansprüche dar. ${ }^{33}$

\section{Vom idealistischen Handlungsethos zur normalisierten Realpolitik}

Getragen vom Glücksgefühl der Vereinigung in Frieden und Freiheit zielte die Bundesregierung in den ersten zwei bis drei Jahren des Untersuchungszeitraums darauf ab, das System der Staatenkonkurrenz unter anarchischen Bedingungen in ein System der verregelten Vergemeinschaftung zu überführen. An die Stelle des Rechts des Stärkeren sollte die Herrschaft des Rechts in einem System kollektiver Sicherheit und Ordnung treten. Die fortschreitende Preisgabe eines solidarischen Handlungsethos im Laufe der 1990er Jahre liegt der von anderen beobachteten Forderung nach "größerer Bedeutung « für sich selbst (Miskimmon/Paterson 2006: 29) und der Idee, die eigenen Interessen genauso durchzusetzen, wie andere das auch machen (Hellmann et al. 2006: 227) zugrunde. In der Gesamtschau lässt sich feststellen, dass bis Ende der 1990er Jahre die Vision von der unteilbaren Verantwortung der Völker als normativer Leitidee - als Antwort auf die Frage, wie die Welt sein sollte - zwar erhalten blieb. ${ }^{34}$ Doch die erklärenden Formeln, wie die Welt tatsächlich strukturiert sei, bezogen sich immer stärker auf das alte Konzept der Machtverteilung und des Rechts des Stärkeren. ${ }^{35}$ Als Reaktion auf die bestehende Lücke zwischen Wunschvorstellung und perzipierter Realität orientierte die Bundesregierung die eigenen normativen Zukunftsprojektionen zusehends an den

$32 »$ Deutschland richtet seine sicherheitspolitischen Strukturen hierauf aus und ist bereit, einen seiner Größe und Bedeutung angemessenen Beitrag zur Stärkung der internationalen Sicherheit zu leisten« (Koalitionsvertrag 2005: 131-132).

33 »Präsident Putin hat in seiner Jahrespressekonferenz in der vergangenen Woche erklärt, dass Nichtregierungsorganisationen in der russischen Gesellschaft eine wichtige Rolle spielen, nicht zuletzt auch deshalb, weil sie das staatliche Handeln und die Machtorgane kontrollieren. Die Botschaft ist wichtig, aber wir sind nicht blauäugig und deshalb erwarten wir natürlich auch, dass sich diese Haltung in der Praxis im Umgang mit den Nichtregierungsorganisationen widerspiegeln wird« (Steinmeier 2006).

34 "Es ist das Konzept der Gleichberechtigung großer und kleiner Staaten, der Machtbeschränkung durch Souveränitätsübertragung an Gemeinschaftsorgane, der wirtschaftlichen Interessenvernetzung und der regionalen Solidarität und der Interdependenz« (Genscher 1990).

$35 »$ Die große Hoffnung, dass nach dem Ende des Ost-West-Konflikts endlich die Ära der multilateralen Kooperation und der weltweiten Herrschaft des Rechts anbricht, ist bisher nicht Wirklichkeit geworden« (Fischer 1999a). 
»machtpolitischen Realitäten«. Statt an den Leitbildern der Herrschaft des Rechts, der Gleichberechtigung aller Staaten, der internationalen Solidarität, des Souveränitätstransfers auf internationale Organisationen und der Transformation von Macht in Recht festzuhalten, veränderte sich die Haltung der Bundesregierung in dieser Frage schleichend. In die Blaupausen für die zukünftige Entwicklung der internationalen Beziehungen wurde immer häufiger die hartnäckig wirksame Logik der Machtverteilung und des Rechts des Stärkeren integriert. ${ }^{36}$ Maull erklärt die Veränderungen in der deutschen Außenpolitik als Resultat eines relativen und absoluten Verlustes an Ressourcen, die zu einer Aushöhlung der Substanz der deutschen Zivilmacht geführt habe, so dass es sich nun um eine »unsichere Macht« trotz kontinuierlicher Zivilmachtsrolle handele.

Die vorliegenden Rekonstruktionen legen jedoch stattdessen nahe, dass die Erklärung für die Veränderungen der deutschen Außenpolitik auf der Ebene der Handlungsregeln zu suchen ist - also das grundlegende Skript von Rolle und Identität deutscher Außenpolitik berührt ist. Die grundlegenden Handlungsregeln deutscher Außenpolitik orientieren sich nicht mehr länger zuvorderst am Motiv der Herrschaft des Rechts, sondern am Recht des Stärkeren entlang machtzentrierter Konzepte des klassischen Realismus. Maull stützt seine Kontinuitätsthese darauf, dass die Bundesregierung am Konzept des never alone festhalte und den Einsatz von Gewalt durch gemeinsame Normen legitimieren würde (Maull 2006b: 274). Dabei ist das Konzept des never alone für sich genommen noch kein überzeugendes Kriterium, da ihm die Bildung einer »Koalition der Willigen« ohne UN-Mandat ebenfalls genüge tun würde. Auch der Verweis, Deutschland habe seine Streitkräfte immer nur aufgrund gemeinsamer Normen eingesetzt, also aufgrund von Bündnissolidarität oder zur Verhinderung von Massenmord, erweist sich angesichts der vorliegenden Rekonstruktionen als problematisch, da an die Stelle des Versuchs den Weltfrieden zum Wohle der Menschheit gemäß dem Leitmotiv »Frieden für alle« zu fördern, das Konzept der »Sicherheit vor den Anderen« in einer gespaltenen Welt zum Wohle und Schutz eng definierter nationaler (und europäischer) Interessen getreten ist. Der Hinweis, Deutschland würde die Bundeswehr weiterhin als politisches und nicht als militärisches Instrument begreifen (Maull 2006b: 274), erscheint zudem als Begründung für die Kontinuität einer zivilmächtigen deutschen Außenpolitik wenig beizutragen, da der Sinn dieser Unterscheidung - auch ohne Rückgriff auf Clausewitz - fraglich erscheinen muss. Genauso wenig kann der Verweis auf die Fortsetzung der deutschen Integrationspolitik und des Souveränitätstransfers (Maull 2006b: 274) als Beleg für Maulls Zivilmachtbefund dienen, da ja

36 »In der multipolaren Welt des 21. Jahrhunderts muss die Europäische Union deshalb zum eigenständigen, politisch handlungsfähigen Subjekt werden« (Fischer 1999b). »Wirksamer Multilateralismus zur Lösung weltumspannender Probleme wie Umweltschutz, Rüstungskontrolle, Diamanten- und Waffenhandel wird im vor uns liegenden Jahrhundert ein konstruktives Mitwirken auch weiterer Staaten voraussetzen. Die Beziehungen zu aufkommenden Schlüsselakteuren wie China, Japan, Südafrika oder Brasilien werden deshalb für uns an Bedeutung gewinnen. Dies gilt besonders auch für das säkulare demokratische Indien, das ein wichtiger Stabilitätsanker in Südasien, einer der gefährlichsten Krisenregionen der Erde, bleibt« (Fischer 2000b). 
gezeigt werden konnte, dass Integration und Souveränitätstransfers eben gerade nicht exklusive Elemente einer genuinen Zivilmachtsorientierung darstellen, sondern auch ein integraler Bestandteil der am nationalen Interesse, der Steigerung des nationalstaatlichen Gestaltungspotentials und dem Recht des Stärkeren orientierten deutschen Weltordnungspolitik sind. Angesichts dieser Entwicklung davon auszugehen, deutsche Außenpolitik sei auch als »unsichere Macht« weiterhin maßgeblich von den Prinzipien und normativen Richtlinien einer Zivilmacht geprägt (Maull 2006b: 273), beziehungsweise es handele sich bei den Veränderungen bloß um "Modifikationen « des kontinuierlich wirksamen Zivilmachtsrollenkonzepts (Harnisch/Maull 2001: 143), erscheint zusehends problematisch. Auch Maull (2011) selbst weist inzwischen auf diese schleichende Entwicklung in einem jüngeren Beitrag pointiert hin.

Mit Blick auf die These Hellmanns von der machtpolitischen Resozialisierung deutscher Außenpolitik, die auch als Versuch interpretiert werden kann, die dominante Behauptung vom Fortbestand der deutschen Zivilmachtsrolle kritisch zu hinterfragen, ergeben sich angesichts der vorliegenden Befunde einige Anknüpfungspunkte. Der für Hellmanns Argumentation zentrale »Machtpolitikresistenztest«, der danach fragt, ob »außenpolitische Initiativen internationale Regelwerke stärken (also die Bindungswirkung internationaler Normen erhöhen) und damit die Spielräume für einzelstaatliche Eigenmächtigkeiten einschränken« (Hellmann 2004: 82) oder nicht, erweist sich insofern als ergänzungsbedürftig, als dort eine generelle Trennung von Machtpolitik einerseits und der Stärkung internationaler Regelwerke andererseits angenommen wird. Dies berücksichtigt wohl insgesamt zu wenig, wie sehr die Bundesregierung Machtpolitik gerade im Rahmen internationaler Regelwerke betreibt, von denen sie annimmt, dass sie diese maßgeblich nach eigenen Werten und gemäß der nationalen Interessen Deutschlands gestalten könne. ${ }^{37}$ Nicht die Stärkung internationaler Regelwerke per se sollte also "getestet« werden, sondern die zugrundeliegenden Begründungsmuster und die daran gebundenen Handlungsziele. Einen solchen Fokus hat Baumann (2006) bereits ganz ähnlich für die Erforschung des deutschen Verständnisses des multilateralen Handlungsstils vorgeschlagen. Gleichzeitig trifft Hellmanns Machtpolitikresistenztest dennoch den neuralgischen Punkt, da er letztlich Außenpolitik daran bemessen sehen möchte, inwiefern sie konsequent am Ideal der Herrschaft des Rechts und der internationalen Solidarität festhält, anstatt das Recht des Stärkeren und die eigenen nationalen Interessen zur Richtschnur des eigenen Handelns zu erklären. Der später von Hellmann selbst reformulierte generelle Befund, der nunmehr statt von der machtpolitischen Resozialisierung von einer »Renaissance machtpolitischer Selbstbehauptung in der deutschen Außenpolitik« spricht (Hellmann 2007: 454), die er als »Wanderung von einem zivilisierungspolitischen zu einem machtpolitischen Pol« versinnbildlicht (Hellmann 2007: 461), weist große Ähnlichkeit mit den hier vorgelegten Befunden

37 »Die Bereitschaft zu substanziellen Beiträgen und das Engagement in allen Gremien wie auch in der konkreten Arbeit der VN in den Krisengebieten wahrt und verstärkt Deutschlands Einfluss auf die künftige Rolle der Weltorganisation« (VPR 2003). 
der Entidealisierung, der Ernüchterung und des Rückgriffs auf die Konzepte des klassischen Realismus auf. Im Kern der hier rekonstruierten Entidealisierungstendenz steht ja die Tatsache, dass eine angesichts der Diskrepanz von gewünschtem Welt-Soll-Zustand und realiter vorgefundenem Welt-Ist-Zustand ernüchterte deutsche Außenpolitik verstärkt auf realistische Konzepte als Antwort auf ein enttäuschendes Weltgeschehen zurückgreift. Allerdings unterstützt die hier vorgenommene Rekonstruktion nicht den Eindruck, diese Entwicklung sei in der zweiten Amtszeit von Rot-Grün »auffallend forciert« worden (Hellmann 2007: 454). Vielmehr passte die Bundesregierung die eigenen normativen Zukunftsprojektionen während des gesamten Untersuchungszeitraums als Reaktion auf die bestehende Lücke zwischen der eigenen idealistischen Wunschvorstellung und der perzipierten Realität zusehends an die vermeintlichen »machtpolitischen Realitäten « an (ähnlich bei Göler 2004: 139). An die Stelle eines grundlegenden Bekenntnisses zur Zivilisierung der Weltpolitik - im Zweifel auch zu Lasten enger definierter deutscher Interessen - trat eine von Effizienzerwägungen gelenkte Fixierung auf den eigenen Vorteil und die eigene relative Machtposition. Die solcherart entidealisierte deutsche Außenpolitik bereitete sich auf die künftig immer stärker werdende Interessenkonkurrenz der großen Mächte in einem multipolar konfigurierten Weltsystem vor, anstatt weiterhin an die Möglichkeit einer vergemeinschafteten Kooperation der Weltgemeinschaft zu glauben. An die Stelle des Leitbilds der Transformation von Macht in Recht und des Prinzips der gleichberechtigten Teilhabe aller Staaten an der neuen kooperativen Weltordnung auf solidarischer Basis und unabhängig von deren Machtpotentialen trat die Idee, die Geschicke der Welt würden künftig durch die Kooperation und Konkurrenz einiger weniger besonders mächtiger Pole bestimmt. ${ }^{38}$

\section{Fazit und Ausblick: Kontinuität, Wandel oder beides zugleich?}

Im Expertendiskurs setzten sich die Kontinuitätsbefunde schon relativ früh nach der Vereinigung immer stärker gegenüber den Wandelprognosen realistischer Ansätze durch. So überwog für Monika Medick-Krakau am Ende der 1990er Jahre im Großen und Ganzen besehen das Kontinuitätsmoment in der deutschen Außenpolitik. Allerdings räumte sie ein, dass sich neben »inkrementalistischer Anpassung « in einigen Feldern auch ein Wandel »mittleren Grades« feststellen lasse (Medick-Krakau 1999: 20). Helga Haftendorn (2001: 444) stellte wenige Jahre später noch immer eine »bemerkenswerte Kontinuität in der deutschen Außenpolitik« fest und noch gut anderthalb Jahrzehnte nach der Vereinigung gelangt Maull (2006c: 1) zu dem Resultat, dass deutsche Außenpolitik paradoxerweise trotz der epochalen Um-

38 »Der ganze Kontinent kann heute nach dieser europäischen Idee leben. Aber die Situation in Bezug auf andere Kontinente hat sich verändert. Europa muss sich aus sich selbst heraus begründen und zeigen, dass es in einer Welt größeren Wettbewerbs, in einer global transparenten Welt Politik nach seinen Wertvorstellungen gestalten kann« (Bundesregierung 2006). 
brüche im Zuge der Vereinigung kontinuierlich am vorgängigen Kurs festgehalten habe.

Diese Befunde kontrastieren deutlich mit den Ergebnissen der diesem Beitrag zugrundeliegenden Analyse. Die Rekonstruktion machte deutlich, dass sich deutsche Außenpolitik im Laufe der 1990er Jahre zusehends entidealisierte und die bis zum Anfang der 1990er Jahre wirkmächtigen Zivilmachtsorientierungen im Zuge dieses Ernüchterungsprozesses zugunsten klassischer Konzepte wie nationales Interesse und Recht des Stärkeren an handlungsleitendem Einfluss einbüßten. Da sich, wie gezeigt wurde, die deutsche Außenpolitik bei der Ausgestaltung internationaler Normen an ihren eigenen enger definierten und nicht an allgemeinen Interessen orientierte, genügte sie den hohen normativen Ansprüchen - wie sie die Definition Maulls an eine Zivilmacht anlegt - nicht. ${ }^{39}$ Auch im Blick etwa auf die deutsche Sicherheitspolitik ergab die Analyse, dass die Ausrichtung an den eigenen nationalen Wertvorstellungen und den damit verbunden Interessen die einzigen identifizierbaren Kontinuitätselemente darstellte - samt des Fortbestehens einer damit eng verbundenen Stabilitätsorientierung.

Jenseits davon fand im Laufe des Untersuchungszeitraums ein alle Dimensionen deutscher Außenpolitik durchdringender Wandlungsprozess statt. So gelten inzwischen Militäreinsätze im Rahmen der doppelt entgrenzten deutschen Sicherheitspolitik als Weltordnungspolitik unter dem Leitbild der »Armee im Einsatz« längst als selbstverständliches Tauschobjekt für internationales Prestige und Gestaltungsansprüche. Die Befunde der verschwimmenden Trennung von Innen und Außen, der zunehmenden Reklamation eines Interventionsanrechts für einige wenige besonders mächtige Staaten und die Überzeugung, das Völkerrecht könne entlang der eigenen Vorstellungen und Werte zunächst in Krisenfällen gebeugt und dann umgeschrieben werden, ergänzen das Bild der drastisch veränderten Identität deutscher Außenpolitik. $\mathrm{Zu}$ dieser neuen Identität gehört der Wandel des Verantwortungskonzepts weg von der Idee der Verpflichtung hin zur Reklamation von Mitsprache- und Gestaltungsanrechten aufgrund des eigenen Potentials. Hinzu tritt die Idee, die Erinnerung an die eigene Geschichte solle nicht mehr länger handlungsbeschränkend wirken, sondern als Grundlage für Führungsansprüche dienen. Das Ende der deutschen Zivilmacht wird aber besonders durch die fortschreitende Preisgabe eines solidarischen Handlungsethos der internationalen Beziehungen deutlich. Statt weiterhin konsequent die Herrschaft des Rechts und die unteilbare Verantwortung der Staaten und Völker unabhängig von Macht und Gewicht der einzelnen Mitglieder der Weltgemeinschaft zu unterstützen, folgt die deutsche Außenpolitik inzwischen wieder unübersehbar einem realistischen Handlungsskript, in dessen Kern die Machtverteilungserwägungen, das Primat des Rechts des Stärkeren und die Ablösung des »reflexive multilateralism« durch einen flexiblen, effektiven multilatera-

39 Maull (1992: 273) formuliert als ein Element der Zivilmachtsidentität »die Bereitschaft der privilegierten westlichen Industriestaaten, Normen zu entwickeln, die allgemeinen Interessen, nicht ihren Sonderinteressen verpflichtet sind, ihr eigenes Verhalten an diesen allgemeinen Prinzipien messen zu lassen und schließlich Anpassungslasten überproportional selbst zu übernehmen, anstatt sie auf Schwächere abzuwälzen«. 
lism by demand in einer aufkommenden multipolaren, das heißt von Mächtekonkurrenz geprägten Weltordnung stehen.

Insgesamt muss es als äußerst erstaunlich gelten, wie sehr das Profil der deutschen Außenpolitik inzwischen jene Kultur der Zurückhaltung abgelegt hat, von der Katzenstein (1997: 2-3) vor gerade einmal fünfzehn Jahren schrieb, sie sei das charakteristischste Elemente deutscher Außenpolitik. Angesichts der hier präsentierten Befunde deutet also einiges darauf hin, dass sich die frühere Kultur der $\mathrm{Zu}$ rückhaltung im fortgeschrittenen Stadium der Auflösung befindet. Die teils vehementen Reaktionen früherer Gestalter deutscher Außenpolitik auf jüngere Entwicklungen machen deutlich, dass auch diese den hier präsentierten Befund einer nicht allein im »Stil«, sondern auch in der »Substanz« deutlich gewandelten deutschen Außenpolitik teilen (Kohl 2011; Fischer 2011).

Die hier dargelegten Befunde kontrastieren teilweise erheblich mit anderen Thesen, die im Forschungsstand vertreten werden. Dort heißt es unter anderem Kontinuität bliebe »die Handlungsmaxime des vereinten Deutschland« (Meiers 2006: 351), oder es wird von der »konsequenten Fortführung des sicherheitspolitischen Kurses « gesprochen (Hilz 2005: 366) beziehungsweise angenommen, dass »entgegen der verbreiteten Auffassung, nach der Wiedervereinigung sei eine weitreichende Neuorientierung des Ziel-Mittel-Komplexes deutscher Außen- und Sicherheitspolitik erforderlich geworden« die »These von einer weitgehenden Kontinuität im auswärtigen Verhalten Deutschlands« zu vertreten sei (Gareis 2005: 46).

Diese Diskrepanz mag dem Umstand geschuldet sein, dass die hier nachgezeichnete Entwicklung, wenn auch langsam, trotzdem aber stetig, vor allen Dingen aber in kontinuierlichen Bahnen und nicht als Ergebnis sprunghafter Ereignisse verlief. Der Wandel stellt sich dabei auch als weitgehend unabhängig von den Regierungswechseln der Jahre 1998 und 2005 dar. Als besonders stabil erweist sich außerdem eine durch ökonomische Interessen legitimierte grundlegende Stabilitätsorientierung der Bundesregierungen, die möglicherweise leicht mit einem Kontinuitätskurs verwechselt werden kann. Das bedeutet nicht, dass die deutsche Außenpolitik nach 1990 bis zum Jahr 2007 keinerlei Kontinuitätselemente aufgewiesen habe. Als Konstanten deutscher Außenpolitik lassen sich das Primat deutscher Interessen, das Streben nach größerer relativer und absoluter Macht, der Trend zur Ausweitung der exekutiven Handlungsfreiheiten, eine Strategie der Einflussmaximierung Deutschlands auf internationale Organisationen, die Reklamation einer deutschen Führungsrolle innerhalb von EU, NATO und UN sowie das grundlegende Ziel, die vermeintlich überlegenen deutschen Wertvorstellungen langfristig möglichst weltweit durchzusetzen, identifizieren. Die Kontinuität deutscher Außenpolitik besteht also nicht in der konsequenten Orientierung des eigenen Handelns am Rollenskript einer Zivilmacht, sondern in der kontinuierlichen Priorität deutscher Interessen. Der den Expertendiskurs lange Zeit dominierende Tenor von der Kontinuität einer zivilmächtigen deutschen Außenpolitik, den neben Hellmann $(2004,2006,2007)$ und Baumann (2006) inzwischen auch von Bredow (2006: 246-247), Schwarz (2005: 22) und Maull (2011) selbst in Frage zu stellen bereit sind, erscheint auch dem Verfasser angesichts der hier vorgelegten eigenen Interpretation kaum noch plausibel. 
Die in jüngerer Zeit immer wieder eingeforderte und angestoßene Debatte über die Zukunft der deutschen Außenpolitik (vor allem ihrer Sicherheits- als Weltordnungspolitik) steht zwar im Großen und Ganzen noch aus. Dennoch nimmt die Zahl der politikwissenschaftlichen Beiträge zu, die sich mit der »normativen Frage«, welche Grundsätze deutsche Außenpolitik denn anleiten sollten, auseinandersetzen. ${ }^{40}$ Jüngere Ereignisse, wie der folgenschwere Luftangriff im Norden Afghanistans (04.09.2009), der Rücktritt des Bundespräsidenten Horst Köhler aufgrund seiner Äußerungen bezüglich der Motive des deutschen Einsatzes in Afghanistan (31.05.2010) sowie die jüngsten Turbulenzen im Zuge der deutschen Enthaltung zur Resolution 1973 des UNSR betreffend der militärischen Durchsetzung einer Flugverbotszone über Libyen (17.03.2011), signalisieren die besondere Relevanz einer politikwissenschaftlichen, gesellschaftlichen und politischen Debatte über die zukünftigen Ziele und Wege deutscher Außenpolitik.

\section{Literatur}

Bauer, Harry/Brighi, Elisabetta 2009: Pragmatism in International Relations, Abingdon.

Baumann, Rainer 2006: Der Wandel des deutschen Multilateralismus, Baden-Baden.

Berger, Thomas U. 1996: Norms, Identity, and National Security in Germany and Japan, in: Katzenstein, Peter J. (Hrsg.): The Culture of National Security: Norms and Identity in World Politics, New York, NY, 317- 356.

Berger, Thomas U. 1998: Cultures of Antimilitarism: National Security in Germany and Japan, Baltimore, MD.

Bryant, Antony/Charmaz, Kathy 2010: Introduction: Grounded Theory Research: Methods and Practices, in: Bryant, Antony/Charmaz, Kathy (Hrsg.): The Sage Handbook of Grounded Theory, London, 1-28.

Bulmer, Simon/Jeffery, Charlie/Paterson, William E. 2000: Germany's European Diplomacy: Shaping the Regional Milieu, Manchester.

Bundesregierung 1998: Der Bundesminister des Auswärtigen, Dr. Klaus Kinkel, gab in der 224. Sitzung des Deutschen Bundestages am 26. März 1998 zur Öffnung der Atlantischen Allianz für Polen, die Tschechische Republik und Ungarn folgende Erklärung der Bundesregierung ab, in: Bulletin 22/1998.

Bundesregierung 2002: Regierungserklärung von Bundeskanzler Gerhard Schröder zu den Ergebnissen des Europäischen Rates am 12. /13. Dezember 2002 in Kopenhagen vor dem Deutschen Bundestag am 19. Dezember in Berlin, in: Bulletin 103/2002.

Bundesregierung 2004: Europäische Sicherheits- und Verteidigungspolitik (herausgegeben vom Bundesministerium der Verteidigung und dem Auswärtigen Amt, Mai 2004), Berlin.

Bundesregierung 2005: Regierungserklärung von Bundeskanzler Gerhard Schröder zum bevorstehenden Europäischen Rat in Brüssel am 16. und 17. Juni 2005 vor dem Deutschen Bundestag am 16. Juni 2005 in Berlin, in: Bulletin 55/2005.

Bundesregierung 2006: Regierungserklärung von Bundeskanzlerin Dr. Angela Merkel zur Europapolitik vor dem Deutschen Bundestag am 11. Mai 2006 in Berlin, in: Bulletin 44/2006.

Dewey, John 1991: The Public and its Problems, Athen.

40 Für einige Anstöße jüngeren Datums siehe etwa Habermas (2010), Hellmann (2010b), Maull (2010) sowie die Beiträge zum Streitplatz »Deutsche Interessen offen diskutieren!« in WeltTrends, No. 71-75. 
Erb, Scott 2003: German Foreign Policy: Navigating a New Era, London.

Fischer, Joschka 1999a: Rede des Bundesministers des Auswärtigen, Joschka Fischer, vor der 54. Generalversammlung der Vereinten Nationen am 22. September 1999 in New York, in: Bulletin 57/1999.

Fischer, Joschka 1999b: Verhandlungen des Europäischen Parlaments. Sitzung am Dienstag, den 12. Januar 1999, 1. Programm des deutschen Vorsitzes und Lage im Kosovo, in: http://www.europarl.europa.eu/debats/debats?FILE=99-01-12\&LANGUE=DE\&LEVEL $=$ DOC $\&$ GCSELECTCHAP $=1 \&$ GCSELECTPERS $=2 ; 05.03 .2007$.

Fischer, Joschka 2000a: Vom Staatenverbund zur Föderation - Gedanken über die Finalität der europäischen Integration (Rede des Bundesministers des Auswärtigen, Joschka Fischer, am 12. Mai 2000 in der Humboldt- Universität in Berlin), in: Bulletin 29/2000.

Fischer, Joschka 2000b: Rede des Bundesministers des Auswärtigen Joschka Fischer, am 4. September 2000 bei der ersten Konferenz der Leiterinnen und Leiter deutscher Auslandsvertretungen in Berlin, in: Bulletin 53/2000.

Fischer, Joschka 2011: Deutsche Außenpolitik - eine Farce, in: Süddeutsche Zeitung, 24.03.2011.

Franke, Ulrich/Roos, Ulrich 2010: Actor, Structure, Process: Transcending the State Personhood Debate by Means of a Pragmatist Ontological Model for International Relations Theory, in: Review of International Studies 36: 4, 1057 - 1077.

Friedrichs, Jörg/Kratochwil, Friedrich 2009: On Acting and Knowing: How Pragmatism Can Advance International Relations Research and Methodology, in: International Organization 63:4, 701-731.

Gareis, Sven Bernhard 2005: Deutschlands Außen- und Sicherheitspolitik. Eine Einführung, Opladen.

Genscher 1990: Rede des Bundesaußenministers, Hans-Dietrich Genscher, vor der 45. Generalversammlung der Vereinten Nationen in New York, in: Bulletin 115/1990.

Goetz, Klaus H. 1996: Integration Policy in a Europeanized State: Germany and the Intergovernmental Conference, in: Journal of European Public Policy 3: 1, 23-44.

Göler, Daniel 2004: Europapolitik im Wandel, Münster.

Göler, Daniel 2010: Die strategische Kultur der Bundesrepublik - Eine Bestandsaufnahme normativer Vorstellungen über den Einsatz militärischer Mittel, in: Dörfler-Dierken, Angelika/Portugall, Gerd (Hrsg.): Friedensethik und Sicherheitspolitik. Weißbuch 2006 und EKD-Friedensschrift 2007 in der Diskussion, Wiesbaden, 185-199.

Habermas, Jürgen 2010: Wir brauchen Europa! Die neue Hartleibigkeit: Ist uns die gemeinsame Zukunft schon gleichgültig geworden?, in: Die Zeit, 20.05.2010.

Haftendorn, Helga 2001: Deutsche Außenpolitik zwischen Selbstbeschränkung und Selbstbehauptung, Stuttgart.

Harnisch, Sebastian/Maull, Hanns W. 2001: Conclusion: »Learned its lesson well?« Germany as a Civilian Power Ten Years after Unification, in: Harnisch, Sebastian/Maull, Hanns W. (Hrsg.): Germany as a Civilian Power? The Foreign Policy of the Berlin Republic, Manchester, 128-156.

Harnisch, Sebastian/Schieder, Siegfried 2006: Germany's New European Policy: Weaker, Leaner, Meaner, in: Maull, Hanns W. (Hrsg.): Germany's Uncertain Power: Foreign Policy of the Berlin Republic, Basingstoke, 95-108.

Hellmann, Gunther 2002: Deutschland in Europa: Eine symbiotische Beziehung, in: Aus Politik und Zeitgeschichte, B48/2002, 24-31.

Hellmann, Gunther 2004: Wider die machtpolitische Resozialisierung der deutschen Außenpolitik. Ein Plädoyer für offensiven Idealismus, in: WeltTrends 12: 42, 79-88.

Hellmann, Gunther 2006: Lamed Power: Germany and European Integration, in: Hellmann, Gunther (Hrsg.): Germany's EU Policy on Asylum and Defence: De-Europeanization by Default?, Hampshire, 156-184.

Hellmann, Gunther 2007: «...um diesen deutschen Weg zu Ende gehen zu können.«. Die Renaissance machtpolitischer Selbstbehauptung in der zweiten Amtszeit der Regierung 
Schröder-Fischer, in: Egle, Christoph/ Zohlnhöfer, Reimut (Hrsg.): Ende des rot-grünen Projekts. Eine Bilanz der Regierung Schröder 2002-2005, Wiesbaden, 453-479.

Hellmann, Gunther 2009: Beliefs as Rules for Action: Pragmatism as a Theory of Thought and Action, in: International Studies Review 11: 3, 638-662.

Hellmann, Gunther 2010a: Pragmatismus, in: Masala, Carlo/Sauer, Frank/Wilhelm, Andreas (Hrsg.): Handbuch der Internationalen Politik, Wiesbaden, 148-181.

Hellmann, Gunther 2010b: Normativ nachrüsten. Deutschlands Rolle in der Welt und wie sie zu gestalten wäre, Internationale Politik, in: http://www.internationalepolitik.de/ 2010/10/01/normativ-nachrusten/; 01.12.2010.

Hellmann, Gunther 2011: Berlins große Politik im Fall Libyen, in: WeltTrends 19:80, 19-22.

Hellmann, Gunther/Baumann, Rainer/Wagner, Wolfgang 2006: Deutsche Außenpolitik. Eine Einführung, Wiesbaden.

Hellmann, Gunther/Weber, Christian/Sauer, Frank 2008: Die Semantik der neuen deutschen Außenpolitik. Eine Analyse des außenpolitischen Vokabulars seit Mitte der 1980er Jahre, Wiesbaden.

Hellmann, Gunther/Wolf, Reinhard/Schmidt, Siegmar 2007: Deutsche Außenpolitik in historischer und systematischer Perspektive, in: Schmidt, Siegmar/Hellmann, Gunther/Wolf, Reinhard (Hrsg.): Handbuch zur deutschen Außenpolitik, Wiesbaden, 15-46.

Hesse-Biber, Sharlene Nagy 2010: Teaching Grounded Theory, in: Bryant, Antony/Charmaz, Kathy (Hrsg.): The Sage Handbook of Grounded Theory, London, 311-338.

Hildenbrand, Bruno 2004: Gemeinsames Ziel, verschiedene Wege. Grounded Theory und Objektive Hermeneutik im Vergleich, in: Sozialer Sinn 5: 2, 177-194.

Hildenbrand, Bruno 2006: Wider die Sippenhaft, in: Sozialer Sinn 7: 1, 159-167.

Hilz, Wolfram 2005: Europas verhindertes Führungstrio. Die Sicherheitspolitik Deutschlands, Frankreichs und Großbritanniens in den Neunzigern, Paderborn.

Janning, Josef 2006: Europäische Union und deutsche Europapolitik, in: Schmidt, Siegmar/ Hellmann, Gunther/Wolf, Reinhard (Hrsg.): Handbuch zur deutschen Außenpolitik, Wiesbaden, 747-762.

Katzenstein, Peter J. 1997: United Germany in an Integrating Europe, in: Katzenstein, Peter J. (Hrsg.): Tamed Power. Germany in Europe, Cornell, 1-48.

Kelle, Udo 1995: Theories as Heuristic Tools in Qualitative Research, in: Maso, Ilja/Atkinson, Paul A./Delamont, Sara/Verhoeven, Jef C. (Hrsg.): Openness in Research: The Tension between Self and Other, Assen, 33-50.

Keller, Reiner 2004: Diskursforschung. Eine Einführung in die Sozialwissenschaften, Opladen.

Kinkel 1995: Deutschland und Japan - Verantwortung in einer Welt im Wandel, Rede von Bundesaußenminister Dr. Kinkel in Tokio vor der Yomiuri International Economic Society am 1.11.1995, in: Bulletin 91/1995.

Kinkel 1998: Der Bundesminister des Auswärtigen, Dr. Klaus Kinkel, hielt anläßlich der Vortragsreihe der Deutschen Gesellschaft für Auswärtige Politik e.V. (DGAP) zum Thema Reden zur Außenpolitik der Berliner Republik am 1. Juli 1998 in Berlin, in: Bulletin $51 / 1998$.

Knapp, Manfred 2004: Die Außenpolitik der Bundesrepublik Deutschland, in: Knapp, Manfred/Krell, Gert (Hrsg.): Einführung in die Internationale Politik, München, 135-200.

Koalitionsvertrag 2005: Gemeinsam für Deutschland. Mit Mut und Menschlichkeit. Koalitionsvertrag von CDU, CSU und SPD vom 11.11.2005, in: http://www.bundesregierung.de/Content/DE/_Anlagen/koalitionsvertrag.html;08.07.2006.

Kohl, Helmut 2011: Wir müssen wieder Zuversicht geben, in: Internationale Politik 16: 5 , 10-17.

Krippendorff, Ekkehart 2010: Für einen deutschen Pazifismus, in: Blätter für deutsche und internationale Politik, 55: 7, 91-97.

Le Gloannec, Anne-Marie 2004: The Unilateralist Temptation: Germany's Foreign Policy after the Cold War, in: Internationale Politik und Gesellschaft 6: 1, 27-39. 
Legewie, Heiner/Schervier-Legewie, Barbara 2004: Anselm Strauss im Interview, in: Forum Qualitative Sozialforschung 5: 3, Artikel 22. http://qualitative-research.net/index.php/fqs /article/view/562/1217.

Link, Werner 2004a: Grundlinien der außenpolitischen Orientierung Deutschlands, in: Aus Politik und Zeitgeschichte, B 11/2004, 8. März 2004, 3-8.

Link, Werner 2004b: Vom Elend des »offensiven Idealismus«. Eine Antwort auf Hellmanns $»$ Traditionslinie« und »Sozialisationsperspektive«, in: WeltTrends 12: 43, 47-51.

Longhurst, Kerry 2004: Germany and the Use of Force: The Evolution of German Security Policy 1990-2003, Manchester.

Malici, Akan 2006: Germans as Venutians: The Culture of German Foreign Policy Behavior, in: Foreign Policy Analysis 2: 1, 37-62.

Maull, Hanns W. 1992: Zivilmacht Bundesrepublik Deutschland. Vierzehn Thesen für eine neue deutsche Außenpolitik, in: Europa-Archiv, Folge 10/1992, 269-278.

Maull, Hanns W. 2004: »Normalisierung« oder Auszehrung? Deutsche Außenpolitik im Wandel, in: Aus Politik und Zeitgeschichte, B11/2004, 8. März 2004, 17-23.

Maull, Hanns W. 2006a: Deutsche Außenpolitik in der Krise, in: Jahrbuch Internationale Politik 2003/2004, Oldenbourg, 191-202.

Maull, Hanns W. 2006b: Conclusion: Uncertain Power - German Foreign Policy into the Twenty-First Century, in: Maull/Hanns W. (Hrsg.): Germany's Uncertain Power: Foreign Policy of the Berlin Republic, Basingstoke, 273-286.

Maull, Hanns W. 2006c: Introduction, in: Maull, Hanns (Hrsg.): Germany's Uncertain Power: Foreign Policy of the Berlin Republic, Basingstoke, 1-12.

Maull, Hanns W. 2010: Weltpolitik in der Turbulenz. Schlussfolgerungen für die deutsche Außenpolitik, Internationale Politikanalyse, Berlin.

Maull, Hanns W. 2011: Deutsche Außenpolitik: Orientierungslos, in: Zeitschrift für Politikwissenschaft 21: 1, 93-117.

Mayring, Philipp 2008: Qualitative Inhaltsanalyse: Grundlagen und Techniken, Weinheim.

Medick-Krakau, Monika 1999: Außenpolitischer Wandel: Diskussionsstand - Erklärungsansätze - Zwischenergebnisse, in: Medick-Krakau, Monika (Hrsg.), Außenpolitischer Wandel in theoretischer und vergleichender Perspektive: Die USA und die Bundesrepublik Deutschland, Baden-Baden, 3-31.

Meiers, Franz-Josef 2006: Zu neuen Ufern? Die deutsche Sicherheits- und Verteidigungspolitik in einer Welt des Wandels 1990-2000, Paderborn.

Merkel 2006: Rede von Bundeskanzlerin Angela Merkel anlässlich der ACG/DGAP-Konferenz am 12. Januar 2006 in Washington (verfügbar im Archiv der Deutschen Gesellschaft für Auswärtige Politik), Berlin.

Merkel 2007: Rede von Bundeskanzlerin Dr. Angela Merkel zur Bilanz der deutschen EURatspräsidentschaft vor dem Europäischen Parlament am 27. Juni 2007 in Brüssel, in: Bulletin 71/2007.

Miskimmon, Alister /Paterson, William E. 2006: Adapting to Europe? German Foreign Policy, Domestic Constraints, and the Limitations of Europeanization since Unification, in: Maull, Hanns W. (Hrsg.): Germany's Uncertain Power: Foreign Policy of the Berlin Republic, Basingstoke, 29-46.

Müller, Harald 2006: Germany and the Proliferation of Weapons of Mass Destruction, in: Maull/ Hanns W. (Hrsg.): Germany's Uncertain Power: Foreign Policy of the Berlin Republic, Basingstoke, 49-65.

Oevermann, Ulrich 1991: Genetischer Strukturalismus und das sozialwissenschaftliche Problem der Erklärung der Entstehung des Neuen, in: Müller-Doohm, Stefan (Hrsg.), Jenseits der Utopie. Theoriekritik der Gegenwart, Frankfurt a.M., 267-336.

Oevermann, Ulrich 1996: Konzeptualisierung von Anwendungsmöglichkeiten und praktischen Arbeitsfeldern der objektiven Hermeneutik (Manifest der objektiven hermeneutischen Sozialforschung), unv. Ms., Frankfurt a.M. 
Oevermann, Ulrich 2000: Die Methode der Fallrekonstruktion in der Grundlagenforschung sowie der klinischen und pädagogischen Praxis, in: Kraimer, Klaus (Hrsg.): Die Fallrekonstruktion, Frankfurt a.M., 58-153.

Paterson, William E. 2010: Does Germany Still Have a European Vocation?, in: German Politics 19: 1, 41-52.

Peirce, Charles Sanders 1965: Collected Papers of Charles Sanders Peirce, Volume V Pragmatism and Pragmaticism and Volume VI Scientific Metaphysics (Hartsthorne, Charles/ Weiss, Paul (Hrsg.)), Cambridge.

Peirce, Charles Sanders 1992: How to Make Our Ideas Clear, in: Houser, Nathan/Kloesel, Christian (Hrsg.): The Essential Peirce, Selected Philosohpical Wrtings (Band 1), 124-141.

Pradetto, August 2006: The Polity of German Foreign Policy: Changes since Unification, in: Maull/ Hanns W. (Hrsg.): Germany's Uncertain Power: Foreign Policy of the Berlin Republic, Basingstoke, 15-28.

Reichertz, Jo 2003: Die Abduktion in der qualitativen Sozialforschung, Opladen.

Risse, Thomas 2004: Kontinuität durch Wandel: Eine »neue« deutsche Außenpolitik?, in: Aus Politik und Zeitgeschichte, B11/2004, 8. März 2004, S. 24-31.

Rittberger, Volker 2003: Selbstentfesselung in kleinen Schritten? Deutschlands Außenpolitik zu Beginn des 21. Jahrhunderts, in: Politische Vierteljahresschrift 44: 1,10-18.

Roos, Ulrich 2010: Deutsche Außenpolitik. Eine Rekonstruktion der grundlegenden Handlungsregeln, Wiesbaden.

Rühe, Volker 1993: Die NATO als Fundament der Sicherheitsarchitektur der Zukunft (Rede des Bundesverteidigungsministers auf der Tagung des Ausschusses für Verteidigung und Sicherheit der Nordatlantischen Versammlung, 21.05.1993), in: Bulletin der Bundesregierung 46/1993.

Scharping 1999: Rede des Bundesministers der Verteidigung, Rudolf Scharping, bei der Deutschen Atlantischen Gesellschaft in Bonn- Bad Godesberg am 18. April 1999, in: Bulletin 18/1999.

Schmalz, Uwe 2002: Deutsche Europapolitik nach 1989/90: Die Frage von Kontinuität und Wandel, in: Schneider/Heinrich, Jopp/Mathias/Schmalz, Uwe: Eine neue deutsche Europapolitik? Rahmenbedingungen - Problemfelder - Optionen, Bonn, 15-68.

Schneider, Heinrich 2002: Deutsche Europapolitik: Leitbilder in der Perspektive - Eine vorbereitende Skizze, in: Schneider/Heinrich, Jopp/Mathias/Schmalz, Uwe: Eine neue deutsche Europapolitik?: Rahmenbedingungen - Problemfelder - Optionen, Bonn 2002, 69-131.

Schöllgen, Gregor 2004: Die Zukunft der deutschen Außenpolitik liegt in Europa, in: Aus Politik und Zeitgeschichte, B11/2004 vom 8.3.2004, 9-16.

Schwarz, Hans-Peter 2005: Republik ohne Kompaß. Anmerkungen zur deutschen Außenpolitik, Propyläen, München.

Steinmeier 2006: Rede des Bundesministers des Auswärtigen, Dr. Frank- Walter Steinmeier auf der 42. Münchner Konferenz für Sicherheitspolitik am 5. Februar 2006 in München, in: Bulletin 12/2006.

Stern, Phyliss Noerager 2010: On Solid Ground: Essential Properties for Growing Grounded Theory, in: Bryant, Antony/Charmaz, Kathy (Hrsg.): The Sage Handbook of Grounded Theory, London, 114-126.

Strauss, Anselm L. 1987: Qualitative Analysis for Social Scientists, Cambridge.

Strauss, Anselm L. 1993: Continual Permutations of Action, New York, NY.

Strauss, Anselm L. 1994: Grundlagen qualitativer Sozialforschung: Datenanalyse und Theoriebildung in der empirischen und soziologischen Forschung, München.

Strauss, Anselm L./Corbin, Juliet 1998: Basics of Qualitative Research: Techniques and Procedures for Developing Grounded Theory, London.

Strübing, Jörg 2002: Just Do It? Zum Konzept der Herstellung und Sicherung von Qualität in grounded theory-basierten Forschungsarbeiten, in: Kölner Zeitschrift für Soziologie und Sozialpsychologie 54: 2, 318-342. 
Strübing, Jörg 2004: Grounded Theory. Zur sozialtheoretischen und epistemologischen Fundierung des Verfahrens der empirisch begründeten Theoriebildung, Wiesbaden.

Strübing, Jörg 2006: Wider die Zwangsverheiratung von Grounded Theory und Objektiver Hermeneutik, in: Sozialer Sinn, 7: 1, 147-157.

von Bredow, Wilfried 2006: Die Außenpolitik der Bundesrepublik Deutschland. Eine Einführung, Wiesbaden.

VPR 2003: Verteidigungspolitische Richtlinien 2003. Erlassen vom Bundesverteidigungsminister, Peter Struck, am 21. Mai 2003.

Wagener, Martin 2006: Normalization in Security Policy? Deployments of Bundeswehr Forces Abroad in the Era Schröder, 1998-2004, in: Maull/ Hanns W. (Hrsg.): Germany's Uncertain Power: Foreign Policy of the Berlin Republic, Basingstoke, 79-92.

Wagner, Wolfgang/Baumann, Rainer/Bösche, Monika/Hellmann, Gunther 2006: German Foreign Policy in Europe: An Interactionist Framework of Analysis, in: Hellmann, Gunther (Hrsg.): Germany`s EU Policy on Asylum and Defence: De-Europeanization by Default?, Hampshire, 1-28.

Weiss, Moritz 2009: Power and Signals: Explaining the German Approach to European Security, in: Journal of International Relations and Development 26: 12, 317-348.

Wessels, Wolfgang 1999: Zentralmacht, Zivilmacht oder Ohnmacht? Zur deutschen Außenund Europapolitik nach 1989, in: Weilemann, Peter R./Küsters, Hanns Jürgen/Buchstab, Günter (Hrsg.): Macht und Zeitkritik (Festschrift für Hans-Peter Schwarz zum 65. Geburtstag), Paderborn 1999, 389-406.

Wolf, Klaus Dieter 1991: Das neue Deutschland - eine $»$ Weltmacht $\ll$ ?, in: Leviathan, Zeitschrift für Sozialwissenschaft, 20: 2, 247-260.

Wulfert, Manuela 2006: Finalität und Verfassung der Europäischen Union. Unter besonderer Berücksichtigung von Frankreich, Deutschland und Großbritannien (Dissertation im Fachbereich Gesellschaftswissenschaften der Universität Kassel), in: unter:http://deposit.ddb.de/cgibin/dokserv?idn=982417195\&dok_var=d1\&dok_ext=pdf\&filename $=982417195$.pdf.

Zangl, Bernhard/Zürn, Michael 2003: Frieden und Krieg. Sicherheit in der nationalen und postnationalen Konstellation, Frankfurt a.M. 